\title{
Long-term studies of photospheric magnetic fields on the Sun
}

\author{
Alexei A. Pevtsov ${ }^{1,}$, Luca Bertello ${ }^{1}$, Yury A. Nagovitsyn ${ }^{2,3}$, Andrey G. Tlatov ${ }^{4}$, and Valery V. Pipin ${ }^{5}$ \\ ${ }^{1}$ National Solar Observatory, 3665 Discovery Drive, 3rd Floor, Boulder, CO 80303, USA \\ 2 Central Astronomical observatory of Russian Academy of Sciences at Pulkovo, 196140 Saint Petersburg, Russia \\ ${ }^{3}$ St Petersburg State University of Aerospace Instrumentation, Bol'shaya Morskaya ul. 67, 190000 Saint Petersburg, Russia \\ ${ }^{4}$ Kislovodsk Mountain Astronomical Station of Pulkovo Observatory, 357700 Kislovodsk, Russia \\ ${ }^{5}$ Institute for Solar-Terrestrial Physics, PO Box 291, Lermontov st., 126a, 664033 Irkutsk, Russia
}

Received 29 May 2020 / Accepted 18 November 2020

\begin{abstract}
We briefly review the history of observations of magnetic fields on the Sun, and describe early magnetograps for full disk measurements. Changes in instruments and detectors, the cohort of observers, the knowledge base etc may result in non-uniformity of the long-term synoptic datasets. Still, such data are critical for detecting and understanding the long-term trends in solar activity. We demonstrate the value of historical data using studies of active region tilt (Joy's law) and the evolution of polar field and its reversal. Using the longest dataset of sunspot field strength measurements from Mount Wilson Observatory (1917-present) supplemented by shorter datasets from Pulkovo (1956-1997) and Crimean (1956-present) observatories we demonstrate that the magnetic properties of sunspots did not change over the last hundred years. We also show that the relationship between the sunspot area and its magnetic flux can be used to extend the studies of magnetic field in sunspots to periods with no direct magnetic field measurements. Finally, we show how more recent full disk observations of the vector magnetic field can be used to study the long-term (solar cycle) variations in magnetic helicity on the Sun.
\end{abstract}

Keywords: Sun: magnetic fields / sunspots / polar fields / solar cycle / helicity

\section{Introduction}

The Sun is our nearest star, and thus, its activity largely determines the complex processes which collectively are called "space weather" and "space climate". One of the most important ingredients of solar activity is the magnetic field. The magnetic field helps to channel energy through the solar atmosphere. It stores the energy, which is later released in the course of violent eruptive event, flares and coronal mass ejections (CMEs). The restructuring of the magnetic field due to magnetic reconnection may destabilize the system and trigger eruptive events. The magnetic field defines important features such as sunspots, chromospheric filaments, and coronal holes. As a humorous saying attributed to the late Robert (Bob) Leighton, a prominent American astronomer, puts it: "If the sun didn't have a magnetic field, then it would be as boring a star as most astronomers think it is." This article is based on an invited talk given by the first author at the Space Climate 7 Symposium held in Canton Orford, Québec, Canada, July 8-11, 2019. We start from describing the early history of the magnetic field observations on the Sun (Sect. 2). Then, we follow up with the description of the synoptic observations of sunspot field strengths (Sect. 3)

\footnotetext{
*Corresponding author: apevtsov@nso. edu
}

and full disk magnetograms (Sect. 4), the current challenges facing the digitization of historical measurements of magnetic field (Sect. 5), and the notes on creation of the Carrington rotation synoptic charts (Sect. 7). We briefly mention the difficulties of measuring magnetic fields and list numerous cross-calibration studies from different instruments (Sect. 6). At the end, we summarize the results of a number of studies employing long-term synoptic datasets including the evolution of the polar magnetic fields (Sect. 8), polarity and tilt orientation of active regions (Sect. 9), the use of sunspot areas as a proxy for the magnetic flux in sunspots (Sect. 10), and the studies of helicity on the Sun (Sect. 11). Section 12 provides a brief conclusion of this review article.

\section{Discovery and early observations of magnetic fields}

The discovery of magnetic fields on the Sun was made in 1908 by a prominent American astronomer, Hale (1908). The measurements were based on a magneto-optical effect (now called Zeeman effect) discovered in 1896 by the Dutch physicist, Zeeman (1897). In his pioneering paper, Zeeman (1897) 
already hypothesized about possible use of his discovery to probe magnetic fields in stellar objects, and in fact, at that time there were already observations, which in retrospect could have provided a clear indication of Zeeman splitting in sunspots. Figure 1 reproduces Figure 36 from Young (1895) showing the observations of a broadening and splitting of $\mathrm{Na}$ I D1 $5895.93 \AA$ and Na I D2 $5889.95 \AA$ spectral lines taken by C. A. Young in 1870. Although the drawing is somewhat crude, one can still estimate the field strength, using spectral line splitting, known wavelengths for the $D 1$ and $D 2$ lines, and the Landè $g$-factor $\left(g_{\text {eff }} \approx 1.33\right)$. This estimate yields a field strength in the range of $3500-4600 \mathrm{G}$. Cortie (1892) reported a broadening of spectral lines of metals (e.g., Fe, Na, Ca, Ti, Ba) in sunspots. Observations of 90 sunspots taken between 1882 and 1889 all showed a different degree of line broadening in sunspots. For some spectral lines, the broadening varied significantly between sunspots, while some lines consistently showed very small, if any, broadening. Later observations by Cortie (1898) showed a significant broadening in spectral lines of Vanadium (V) in the spectral range of $\lambda$ 6000-6300 $\AA$. Cortie (1904) published a summary of his measurements of line broadening conducted between 1883 and 1901. He noted that for some spectral lines, the line broadening appeared stronger during the period of sunspot maximum as compared to sunspot minimum. The described line broadening is suggestive of Zeeman broadening in sunspots, but at that time no such a connection was made. The line broadening in sunspots was also observed by several other observers (e.g., Smith, 1904), perhaps, even as early as the first observations of spectral lines in sunspots by Lockyer (1867). In the early 1900s line-broadening in sunspots was the subject of extensive studies, and in fact, at the third meeting of the International Union on Cooperation in Solar Research (a predecessor of the International Astronomical Union), the Committee on Sun-Spot Spectra outlined several tasks for collaborative observations to improve the understanding of this phenomenon (Report of Committee on Sun-Spot Spectra, 1908).

In 1904 and 1906, Mitchell (1904, 1906) took observations of what he called "reversed" and "weakened" lines. The example of patterns he observed (see Fig. 2) strongly resembles Zeeman splitting. Mitchell (1905) gives the following striking description of "reversals" in spectral lines of Vanadium $(V)$ : "The lines $\lambda$ 6224.71, 6243.06, 6243.32, and 6252.05 have been seen so widely reversed at times as to give the effect of a pair of hazy lines, rather than a single line split in two." 1

Table 1 in Mitchell (1906) provides an approximate width of the reversal of five observations taken in 1905 and 1906, which allowed to estimate the field strength as follows: Fe I $5250 \AA \approx 2200 \mathrm{G}, \mathrm{Cr}$ I $5781 \AA$ about $3160 \mathrm{G}$, Ti I $6064 \AA$ - 2160 G, Fe I $6137 \AA 2690$ G, and Fe I $6173 \AA \approx 2360 \mathrm{G}$ (Harvey, priv. commun.). These field strengths are similar to modern measurements of field strength observed in sunspots. The reader can verify these estimates by replacing the $s \Delta x$ term in equation (1) from Pevtsov et al. (2019b) with the width of reversal listed in Mitchell (1906) and using appropriate Landé (g) factors. For example, using $0.170 \AA$ reversal width and $g=3.00$ for Fe I $5250.22 \AA$ spectral line yields $\approx 2200 \mathrm{G}$ field strength. Using the line broadening from Cortie (1892) yields weaker field strengths (e.g., about 700 G for Fe I $6173.34 \AA$ A)

\footnotetext{
1 The wavelengths are in $\AA$.
}

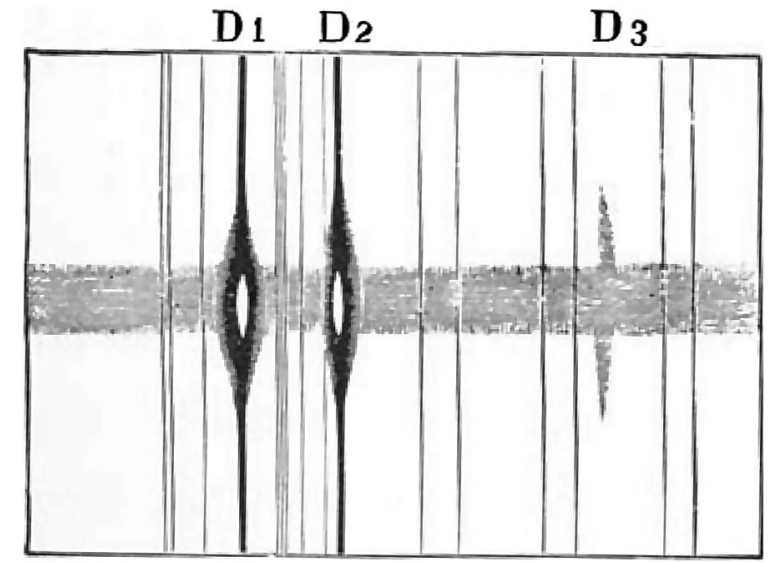

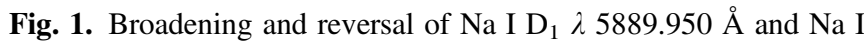
$\mathrm{D}_{2} \lambda 5895.924 \AA$ lines depicted in Figure 36 of Young (1895). Grey horizontal band corresponds to a location of a sunspot on the (vertical) spectrograph slit at the time of observations.

although the spectral line measurements taken in mid-1880s have much larger uncertainties.

In 1905-1906, G.E. Hale attempted detecting the magnetic field in sunspots. His first attempt failed; no expected signature of magnetic field was observed. Meanwhile, the improvements in the sensitivity of the photographic plates in the red part of spectrum (Wallace, 1907) allowed taking spectroheliograms in $\mathrm{H}_{\alpha}$. These new spectroheliograms showed the well-developed whirls around some sunspots. The helical appearance of $\mathrm{H}_{\alpha}$ whirls may imply the presence of the magnetic field, which stimulated Hale to revisit his previous attempt to measure the magnetic field in sunspots. The measurements taken in 1908 were successful in detecting the longitudinal magnetic field in sunspots (Hale, 1908). This paper is dated July 3, 1908. On July 6,1908 , Hale communicated with Zeeman by sending a copy of his paper and two photographic (glass) plates showing the (now called) Zeeman splitting in several spectral lines in the wavelength range of $\lambda 6250-6360 \AA$ (Zeeman, 1913). In his reply, Zeeman suggested additional tests: "One would expect that a change in the direction of rotation in a solar vortex ought to change the sign of a circular polarization; and that a sun spot on the sun's limb ougth to show the lines separated and plane polarization to show the lines separated a plane polarized." On September 26, 1908, Zeeman received a short telegram from Hale: "Vortices rotating opposite direction show opposite polarities; spot lines near limb, plane polarized" (page 132, Zeeman, 1913). Thus, by late 1908 both the longitudinal and transverse fields in sunspots were discovered by Hale.

\section{Synoptic observations of sunspot field strengths}

Hale's early observations made it clear that successful measurements of magnetic field in sunspots require high spatial and spectral resolutions. The construction of a necessary instrument (the 150 feet solar tower telescope) had begun in 1909, and was completed in May 1912 including the Littrow spectrograph (Howard, 1985). On 4 January 1917, Mount Wilson 


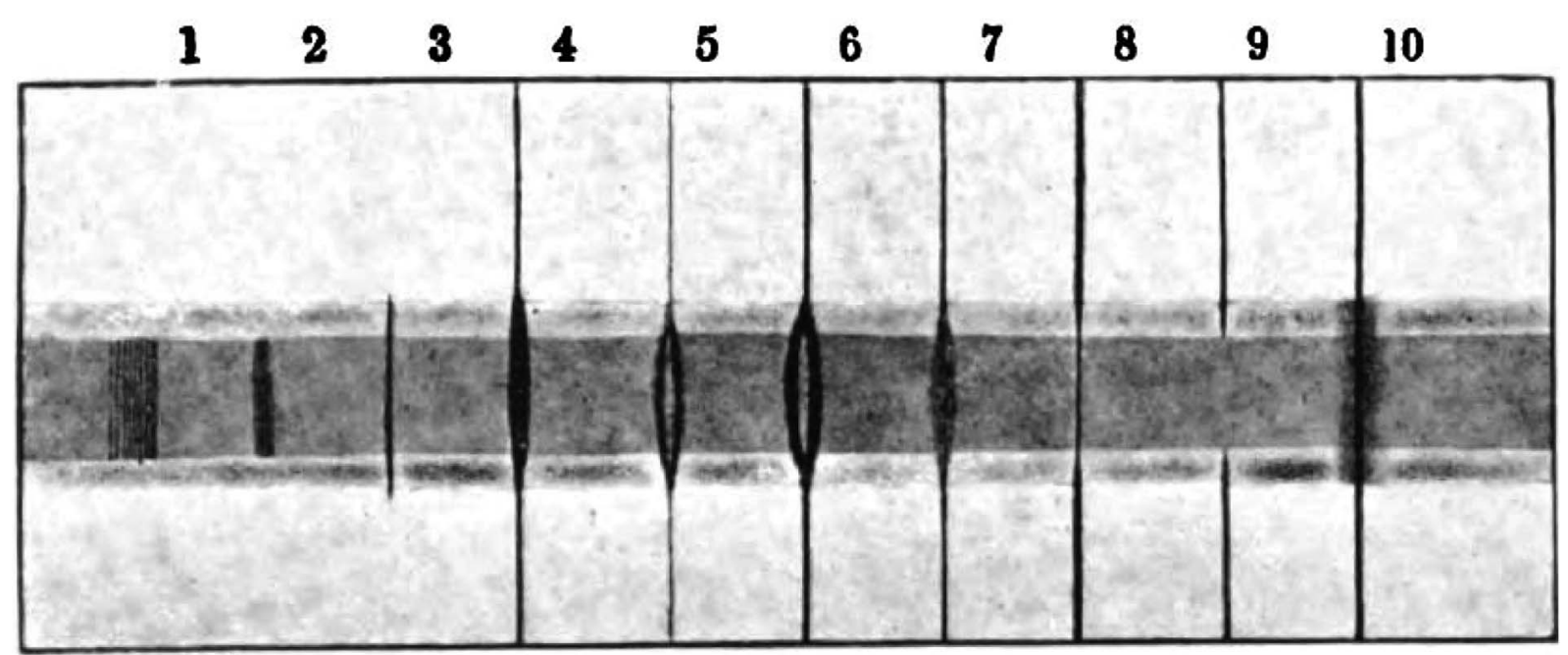

Fig. 2. Examples of spectral line types summarized by Mitchell. Type 4 corresponds to a widened line, types 5-6 are widened lines with the centers reversed bright, and type 7 is widened and weakened line. Grey horizontal band corresponds to sunspot location on a spectrograph slit. Adopted from Report of Committee on Sun-Spot Spectra (1908).

Observatory (MWO) started their synoptic program of daily measurements of sunspot polarities and field strengths. The program has continued till the present day with only minor interruptions mostly due to funding issues. The measurements employ a simple device consisting of a retarder (a quarter-wave plate), a polarizer (e.g., a Nicol prism), and a glass tip plate for measuring the separation between the left/right-hand polarized components resulted from the Zeeman effect in the longitudinal field. The reader can find additional details on the measurements of magnetic field using this technique in Pevtsov et al. (2019b).

In early 1940s, Harald von Klüber of Potsdam Observatory (Germany, now Leibniz Institute for Astrophysics Potsdam, AIP) employed a photographic technique with a circular polarization analyzer for measuring magnetic field in sunspots (von Klüber, 1948). The first observations were taken in 1942 (Staude, 1991). Unlike the single-point measurements at MWO, the photographic image of spectral lines was used to measure the magnetic field in multiple locations across a sunspot, thus allowing for the study of the distribution of magnetic fields in sunspot and even leading to the creation of arguably the first map (a magnetogram) of magnetic field in sunspots (Schröter, 1953). Examples of Potsdam measurements can be found in von Klüber (1948) and Künzel et al. (1956c). Summary of all Potsdam/AIP observations can be found in Grotrian (1953, 1956) and Künzel et al. (1956a-c, 1957). Unlike MWO observations, Potsdam measurements may not include all sunspots that were present on the disk on the day of observations.

In mid-1950s, systematic measurements of magnetic field strength was started in several observatories in the Soviet Union (present day Russia): the Main (Pulkovo) Astronomical Observatory, the Crimean Astrophysical Observatory (CrAO), and the Institute of Terrestrial Magnetism, Ionosphere, and Radio Wave Propagation (IZMIRAN). Later, the measurements were taken at seven observatories, which formed a network spanning over $100^{\circ}$ in longitude, between $30.3262^{\circ} \mathrm{E}$ and $132.1656^{\circ} \mathrm{E}$. The measurement method employed by the Soviet (later Russian) network was similar to the approach used at MWO, where the observer took measurements visually using a tip plate installed in a spectrograph image plane. However, the Kislovodsk Mountain Astronomical Station (KMAS) of Pulkovo Observatory also used a photographic technique where the observer would take a photographic image of the spectra of each spot, and measured the splitting later in the laboratory. Unfortunately, the KMAS observations are not digitized yet and thus will not discussed here.

Daily sunspot polarity and field strength measurements were taken by the Russian groundbased network till 1997. After 1998, the dataset continues using observations from a single station at CrAO. The magnetic field observations from all stations were collected at Pulkovo Observatory and summarized in a monthly Bulletin of "Solar Data" (Russian analog of the "Solar-Geophysical Data"). A cursory examination of these published drawings indicates that the magnetic field measurements of small amplitudes (about $1000 \mathrm{G}$ ) were often not included. The original drawings (which include all measurements) were digitized (tabulated) and are available online as the "Combined Database of Sunspot Magnetic Fields" at http://www.gao.spb.ru/database/mfbase/gindex.html. Observations from CrAO for later periods (1983-2019) are available at https://sun.crao.ru/observations/sunspots-magnetic-field. For additional details about the Russian datasets, the reader can consult Pevtsov et al. (2011).

Figure 3 shows magnetic field strength and polarity for daily observations taken at MWO, CrAO, Potsdam, and Pulkovo Observatory. On average, the mean field strength in sunspots is about $2000 \mathrm{G}$ for all three datasets. However, the scatter around this mean value is larger for $\mathrm{CrAO}$ measurements. Both MWO and CrAO datasets were acquired with the help of many different observers, while at Pulkovo, it was a single observer (Dr. Gennadij F. Vyal'shin) who took the observations from the mid-1950s until he passed away in 1999.

Because of the high latitude location of Pulkovo Observatory $\left(59.77^{\circ} \mathrm{N}\right)$, the daytime observations there are very limited during the winter months (vertical gaps in Pulkovo data). The vertical gaps in $\mathrm{CrAO}$ data correspond to the periods of missing 


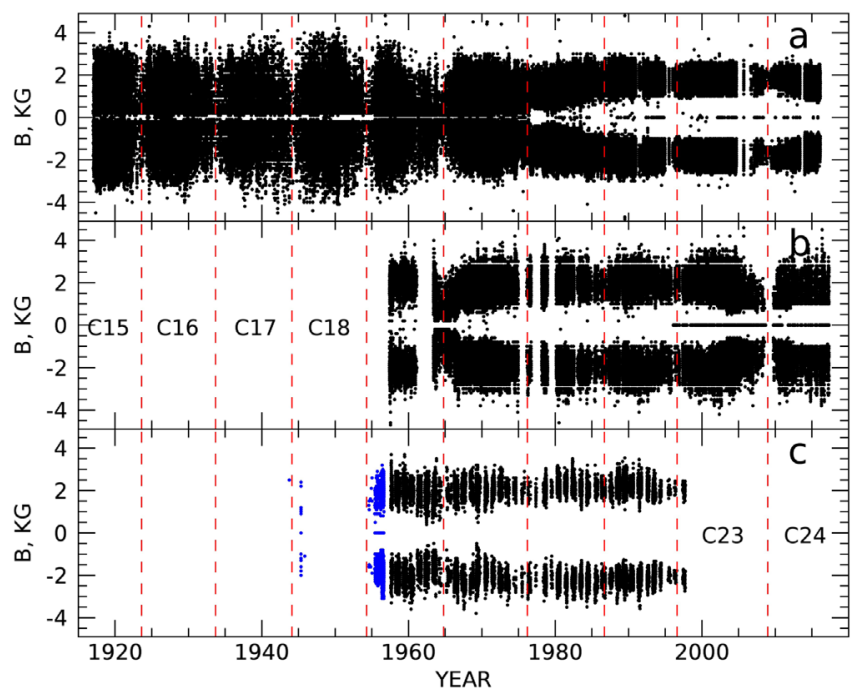

Fig. 3. Daily measurements of field strengths and polarities (dots) from (a) MWO, (Pevtsov et al., 2019a) (b) CrAO, and (c) Pulkovo Observatory. Blue color dots correspond to the Potsdam observations, which have been digitized to date (1945, 1954-1956). Red vertical dashed lines mark approximate time of solar minima. Periods corresponding to solar cycles $15-18$ and 23-24 are marked in panels $\mathrm{b}$ and $\mathrm{c}$.

digital data. The major vertical gaps in MWO dataset corresponds to a period when the observations stopped due to funding issues or when the digitized portion of image did not include the date of observations (Pevtsov et al., 2019b).

All datasets show the solar cycle variations of maximum field strength (see a "wavy" pattern in upper and low "envelops" of the data points, Fig. 3). On average, the maximum field strength of sunspots at the maximum of the solar cycles is larger (about $3500 \mathrm{G}$ ) than the maximum field strength at the cycle minima (about $2500 \mathrm{G}$ ). This "wavy" pattern disappears in MWO data after 1961. In October 1961, the wavelength for sunspot observations at MWO was changed from $\mathrm{Fe} I$ $6173.343 \AA$ to Fe I 5250.22 $\AA$. This change also required installing a thicker tip plate. These two changes resulted in an imposed upper limit on the maximum measured field strength (about $3000 \mathrm{G}$ ). This and other systematic patterns in MWO dataset are described in Pevtsov et al. (2019b).

Another interesting systematic pattern is a gap in weak field strengths. Until about 1978, the measured amplitude of sunspot field strengths at MWO could be as weak as 100 G. After 1978, however, there was a change in the measuring approach, when one of the observers found that under good observing conditions the sunspot field strength is never smaller that about $1000 \mathrm{G}$. All other observers had to "learn" this technique, which may explain a gradual development of gap over the period of several years. Interestingly, CrAO measurements (Fig. 3b) exhibit the development of a similar gap albeit much earlier than at MWO dataset. The gap in weak field strengths was present in early CrAO observations (1957-1962). However when the observations were temporarily moved to different instruments the gap disappeared. In the early 1970s when observations started at the (then new) Tower Solar Telescope, BST-2 (Pevtsov et al., 2011), the gap re-appeared. Potsdam and
Pulkovo observations exhibit a gap in weak fields in sunspot from the the beginning of its datasets (Fig. 3c). Due to a funding shortage, daily observations at MWO were stopped on 15 September 2004 (see two major vertical gaps in Fig. 3a). The observations restarted on 25 January 2007, but under the condition that the magnetic field in smaller spots would not be measured. This could be identified as a hard lower limit in measured fields till about 2010 (see, Fig. 3a around the minimum of cycle 23). After 2011, there appears to be a systematic trend of a declining low limit for the weakest measured field strengths between 2010 and 2016 (last year of the digitized dataset). However, after 2010, all observations at MWO were taken by a single observer (who has been working on a voluntary basis) and one cannot exclude the possibility of a long-term trend in these measurements as an explanation of a steady decline in low envelop of sunspot field strengths. Such longterm drifts are known in sunspot number counts (e.g., Clette et al., 2014). Whether this trend is real or an artifact, needs to be further investigated. Unfortunately, $\mathrm{CrAO}$ observations are not able to help with resolve this question as Figure 3 shows that after February 2012 the lowest measured field strength is limited to $1000 \mathrm{G}$, while in previous years, the field strengths below that limit had been measured.

Despite some non-uniformity in the observations, Figure 3 shows no indication of any long-term changes of the magnetic field strength of sunspots. A trend of the declining field strength in sunspots was first reported by Penn \& Livingston (2006), and later studied in more detail by Penn \& Livingston (2011) and Livingston et al. (2012). Pevtsov et al. (2011, 2014b), however, question the existence of a long-term decline in sunspot field strength based on the analysis of observations from the MWO and the Russian network. In their comparison of measurements from different stations, Pevtsov et al. (2011, 2014b) argued that the measurements of the magnetic field in small sunspots are more likely to be affected by the atmospheric seeing and the observer's experience, while the magnetic fields of large sunspots are less affected. Selecting the sunspots with the largest field strength observed each day significantly improves the agreement between the measurements from different stations. The time series of these sunspots with (daily) "strongest" field strength shows a clear solar cycle variation, but no long-term trend over the last century. The cycle variations are related to the fact that the larger sunspots with stronger magnetic field typically emerge at the maximum of sunspot cycle, while sunspots developing at the beginning and the end of cycle tend to be smaller in size (and thus, weaker in their magnetic field strength). Nagovitsyn et al. (2012) demonstrated that the decline reported by Penn and Livingston could be related to the increase in the number of (measured) small sunspots relative to the number of the large spots with stronger field strengths over the course of the declining phase of solar cycle 23 . Lefèvre \& Clette (2011) found that during cycle 23 there was a deficit of small-size sunspots which they thought could contribute to the apparent decline in measured field strength by Penn and Livingston.

Livingston \& Watson (2015) refuted the earlier findings and concluded that during 2010-2015, the maximum field strength in sunspots does not change.

Another interesting observation is that, despite a significant difference in amplitude of sunspot cycles, the maximum field strength in all cycles does not change too much from one cycle 
to another (see, Figs. 3a and 3b). If one assumes (e.g., Pevtsov et al., 2011; Clette \& Lefèvre, 2012; Nagovitsyn et al., 2012) that sunspots with stronger field strength are formed deeper in the convection zone as compared with their weaker field strength counterparts, the lack of dependence of maximum of sunspot field strength on amplitude of cycle may suggest that the width of the dynamo region in the convection zone, where the sunspot magnetic field is formed does not depend on amplitude of cycle. The maximum field strengths observed in sunspots in early MWO observations are about $5000 \mathrm{G}$, with the strongest measured field strength of $6100 \mathrm{G}$. While these measurements are extremely rare, they might seem larger as compared with the modern magnetographic observations of sunspots. We note, however, that in February 2014, Hinode observations yield a similarly strong sunspot field strength of about $6250 \mathrm{G}$ (Okamoto \& Sakurai, 2018). Moreover, the sunspot with such an extremely strong magnetic field was observed in a declining phase of low-amplitude solar cycle 24 .

The long-term observations conducted over many decades may be non-uniform as the data are taken by many different observers with varying level of experience. Furthermore, the measuring practices could change with time and the development of new knowledge in the field may also affect the observations as one can see from several systematic patterns shown in Figure 3. Pevtsov et al. (2011) used an approach based on selecting the largest sunspot field strength measured by all observatories on a same day. They then normalized the distribution of these field strengths to the mean and standard deviation of a "reference" station. However, this normalization was based on the assumption that the statistical properties of sunspot distributions do not change over the time, which is a priori unknown. The distribution of sunspot field strengths and their areas is discussed later in Section 10. We note that Figure 14 indicates that the distribution of magnetic fluxes in well-developed sunspots does not significantly change during cycles 15-24.

Several past studies found a relatively good agreement between the field strengths measured at MWO, Potsdam, and CrAO (e.g. Steen \&Maltby, 1960; Lozitska et al., 2015), as well as between the manual and the magnetographic observations (e.g., Pevtsov et al., 2019b). As we demonstrate in the following sections, these historical datasets of sunspot field strengths continue to be important for modern-day scientific research. Thus, for example, Lundstedt et al. (2015) used sunspot field strength measurements from MWO in combination with the torus model of a CME to interpret the geomagnetic storm of May 1921. Pevtsov et al. (2016) employed the magnetic field measurements from MWO to construct synoptic maps of magnetic field using non-magnetic observations.

\section{Full disk magnetograms}

Historically, maps of the magnetic field (magnetograms) were taken either over a small area on the sun or over the full disk. Here we review only the full disk observations as they are the most relevant to space climate research.

The first successful measurements of solar magnetic fields using a photoelectric device was done by Thiessen (1946), and the first photoelectric magnetograph was developed by H. W. Babcock in the early 1950s (Babcock, 1953). Initially, this instrument operated at the Hale Laboratory telescope in Pasadena, California. Starting in 1957, observations were made with the magnetograph at the 150-ft tower telescope at MWO. An important aspect relevant to this description is that as a recording device this (first generation) magnetograph used an oscilloscope equipped with a 35-mm photographic camera. Example of such early full disk magnetograms (oscillograms) can be found in Babcock (1953, Fig. 5). The oscilloscope set up was used to record magnetograph signal till end of 1962. During the period 1963-1968 (second generation magnetograph), an analog magnetograph signal was recorded on an $X-Y$ servo plotter display (Howard, 1963). The solar disk was scanned in a raster pattern with a square aperture of $23^{\prime \prime}$ a side until 1967 when it was reduced to $17.5^{\prime \prime}$. The separation between subsequent scans was equal to the aperture size. The $X-Y$ servo plotter pen assembly moved over the plotting paper in the same pattern that the aperture effectively scanned the solar image. Two separately actuated color pens were lowered to write a coded sequence as the magnetic signal varied. Figure 4 shows an example of a "pen-plot" magnetogram, and Figure 5 provides an example of converting the "pen-plots" to the field strengths and the contour maps. In 1965, the instrument was upgraded with a digital data recording system. First observations of this third generation magnetograph were taken in the summer of 1966 (Howard, 1974). With some additional later modifications this full disk magnetograph continued observations through 2013.

In the mid-1960s, a dual channel Babcock-type full disk magnetograph was operating at the Kitt Peak National Observatory in Tucson, Arizona (e.g. Pierce, 1969). The data was recorded on punch paper. In about 1967, the data began being recorded on magnetic tapes. This magnetograph was used until about the mid-1980s when the full disk observations were then transferred to the 40-channel and 512-channel magnetographs. Only some observations from this early instrument have survived and only as photographic images. The scientific value of these data is very limited due to the unknown flux scaling, possible geometric distortions and zero point offset. The polarity information may, however, be of some value.

Observations from the 40-channel magnetograph (19701975, Livingston \& Harvey, 1971; Livingston et al., 1971) started a long-term dataset of full disk magnetograms (or National Solar Observatory/NSO time series). The dataset was continued by the 512-channel magnetograph (1974-1993, Livingston et al., 1976), which was later replaced by NASA/ NSO Spectromagnetograph (SPM, Jones et al., 1992). In 2003, the SPM was replaced by the Vector Stokes Magnetograph (VSM) on Synoptic Optical Long-term Investigations of the Sun (SOLIS) platform (Keller et al., 2003; Balasubramaniam \& Pevtsov, 2011).

In 1976, the Wilcox Solar Observatory (WSO, USA) began taking daily lower resolution observations of full disk longitudinal magnetograms (Scherrer et al., 1977). The idea was inspired by the Academician A. B. Severny (Kotov \& Mozzerin, 1988) of CrAO (P. Scherrer and L. Svalgaard, pers. commun.)

Figure 6 provide examples of full disk magnetograms from MWO, NSO SPM, and WSO. Observations with larger pixel size usually result in a better $S / N$ ratio, but it affects the representation of small scale structures.

The measurement of magnetic fields using the Zeeman effect is typically based on measuring the circular polarization. 


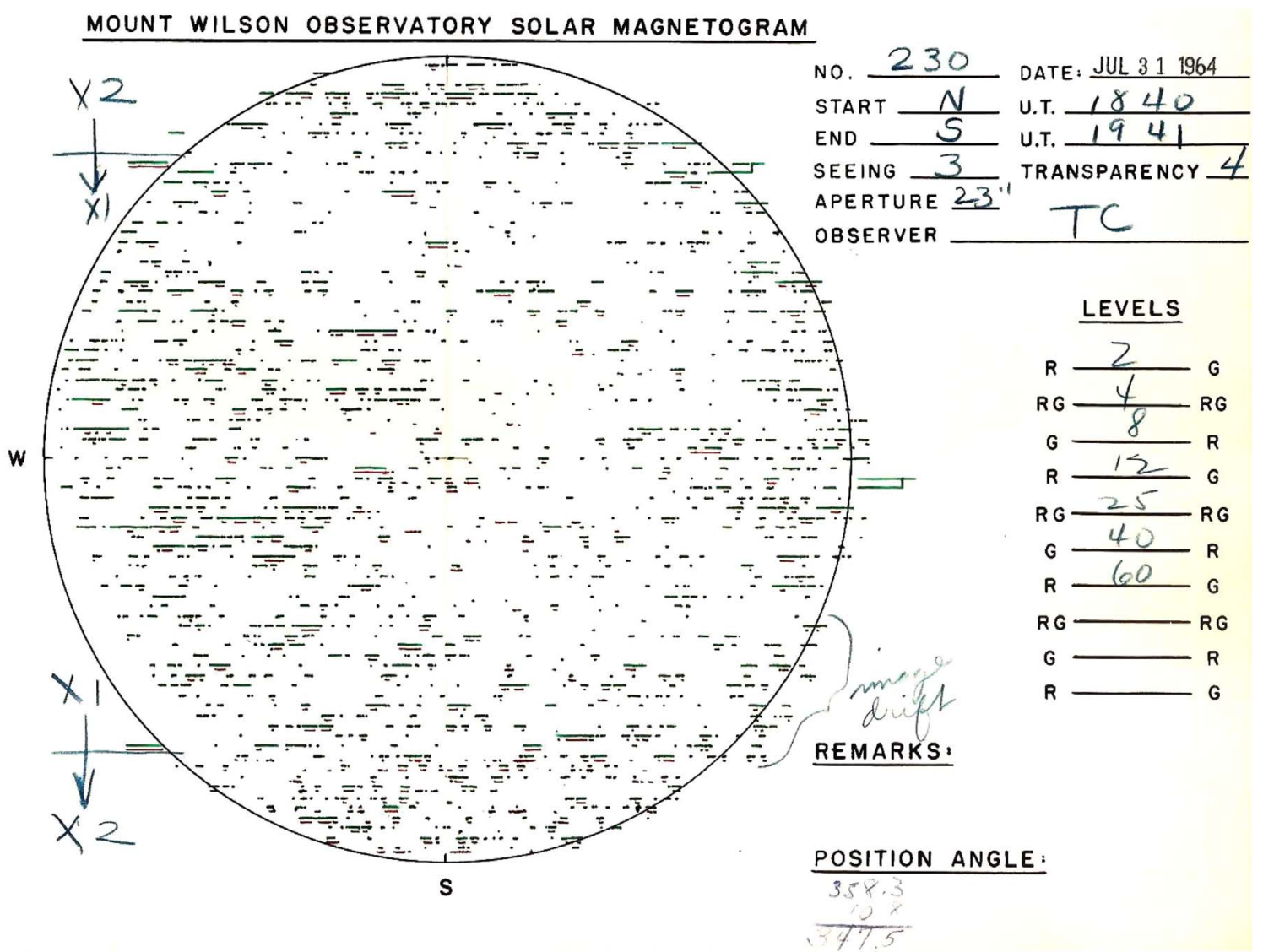

Fig. 4. Example of pen-plot magnetogram taken 31 July 1964. Plotting levels (in units of Gauss) are listed on the right side of image. The extension of lines beyond the solar limb indicate that during some scans, the image drifted off center of the scanning area. Courtesy R. Ulrich.
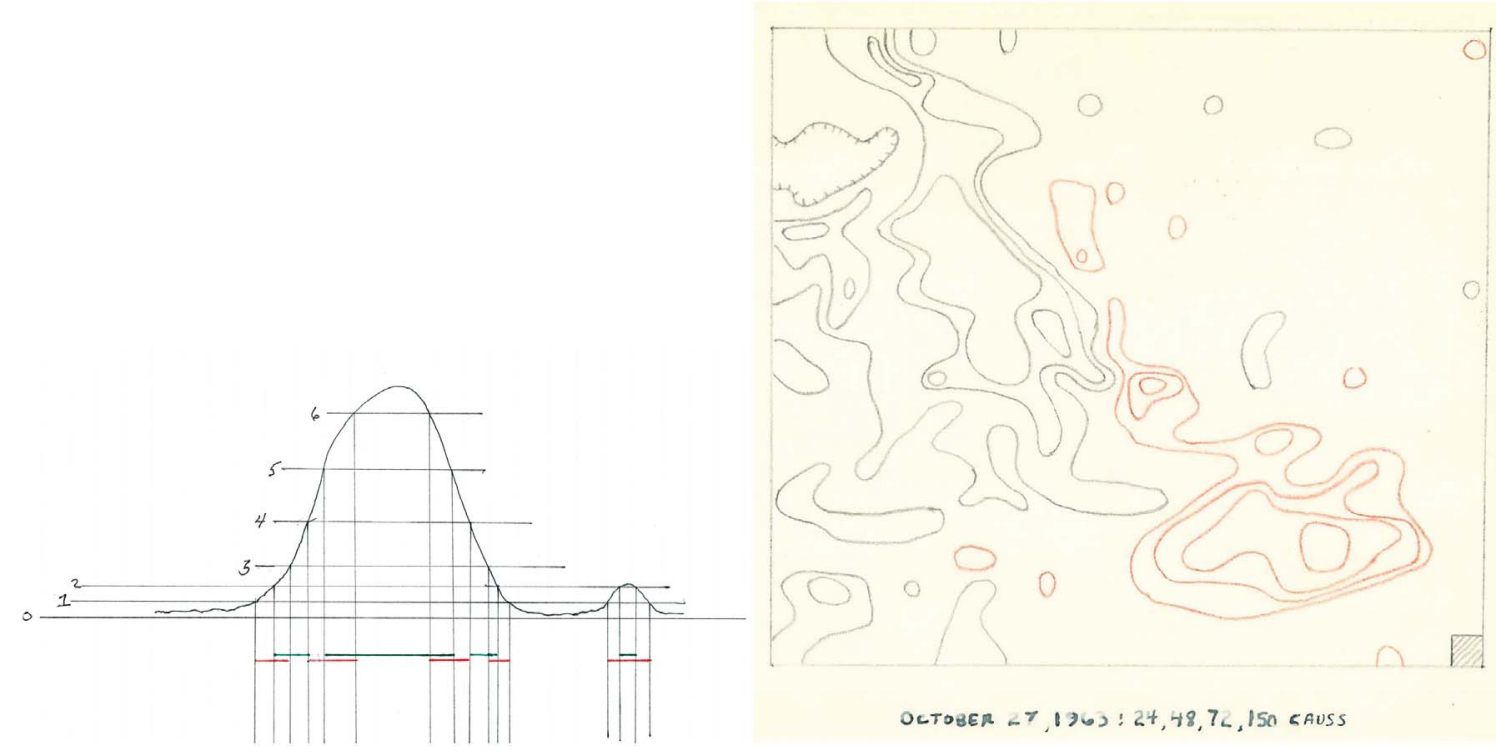

Fig. 5. Left: interpretation of pen-plots in terms of physical units. Each numbered level has a corresponding value in Gauss as indicated in Figure 4 under "Levels". Right: Contour levels (in Gauss) reconstructed from a pen-magnetogram taken on 27 Oct. 1963. Courtesy J. Harvey. 

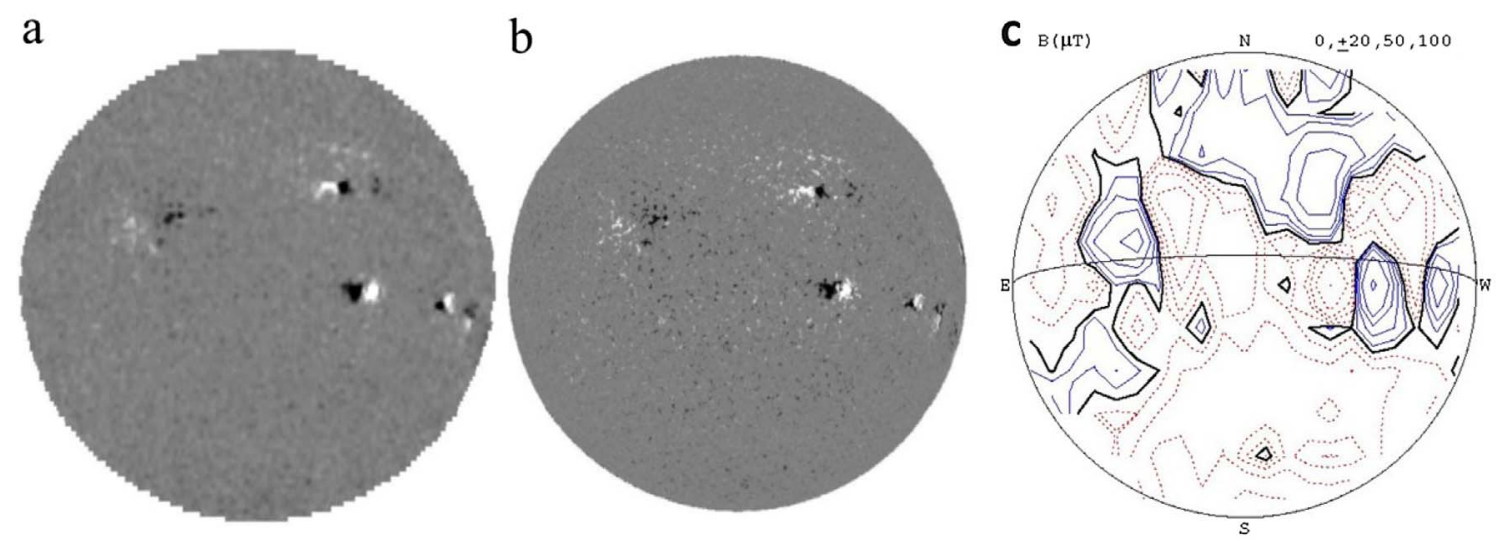

Fig. 6. Example of full disk longitudinal magnetograms taken on 9 March 1994 by magnetographs at (a) MWO, (b) NSO SPM, and (c) WSO. White/black halftones correspond to positive/negative polarity. For display purposes, MWO magnetogram is scaled between $\pm 50 \mathrm{G}$, while NSO SPM is scaled between $\pm 100 \mathrm{G}$. Contours for WSO magnetograms are shown in upper part of panel c.

Due to the technical limitations (type of available detectors and polarization modulators), early instruments employed various simplified techniques. For example, the polarization could be measured only in one or two fixed wavelength bands in a spectral line. The longitudinal (or line-of-sight, LOS) component of the magnetic field $B_{\mathrm{LOS}}$ was then derived as a function of measured polarization. The position of the wavelength band in the spectral line profile was selected to maximize the use of a linear portion of spectral line profile within the range of moderate magnetic field strengths, $B_{\mathrm{LOS}} \propto S_{V} / S_{I}$, where $S_{V}$ and $S_{I}$ represent the intensity of circularly polarized and total measured intensity. However, for stronger magnetic fields, the Zeeman splitting will result in such a position where the core of one of the Zeeman component will move close to the observed wavelength band. This introduces a non-linearity to $B \propto S_{V}$, and in the extreme cases when the magnetic field increases even further, the measured $S_{V}$ will decrease, while the magnetic field continues increasing. This so called magnetic saturation will exhibit itself as a doughnut hole in the middle of some sunspot umbrae with strong magnetic fields. Even later instruments (e.g., Michelson Doppler Imager, MDI on board of Solar and Heliospheric Observatory, SOHO, Scherrer et al., 1995), which measure polarization in a few wavelength bands across the spectral line profile may still exhibit such saturation. Using a simplified approach to measuring magnetic fields may sometimes result in significant differences in the field strengths measured by different instruments. The differences could be due to a correction for the instrumental polarization, or the approach for scaling the measured polarization to the magnetic field. Spatial averaging of unresolved magnetic structures will also lead to difference in measured field strength and a zero level displacement between the instruments with a significantly different pixel size.

By their nature, longitudinal magnetograms do not contain sufficient information to derive the true orientations of the magnetic-field vectors. ${ }^{2}$ The earliest observations of vector

\footnotetext{
${ }^{2}$ However, see Plotnikov \& Kutsenko (2018), who demonstrated that the line profile of Stokes $V$ and $I$ may contain information about both longitudinal and transverse components.
}

magnetic fields in solar active regions were conducted at $\mathrm{CrAO}$ in the early 1960s (Stepanov \& Severny, 1962; Severny, 1965). By the early 1980s, a number of vector magnetographs were developed around the world, with the most prolific instruments operating in Czechoslovakia, East Germany (Pflug \& Grigoryev, 1986), Japan (National Astronomical Observatory of Japan, NAOJ, Ichimoto et al., 1993), the Soviet Union (CrAO, Pulkovo and Sayan observatories), and the USA (NASA's Marshall Space Flight Center/MSFC, Mees Solar Observatory of University of Hawai'i, High Altitude Observatory/HAO) (for review, see individual articles in Hagyard, 1985; Cacciani et al., 1990). By the mid 1990s, the most important vector magnetographs in operation were NASA's MSFC magnetograph (Hagyard et al., 1982), the Advanced Stokes Polarimeter (ASP, Skumanich \& Lites, 1987), the Haleakala Stokes Polarimeter (HSP, Mickey, 1985), the Okayama Astrophysical Observatory (OAO, Makita et al., 1985a), the Sayan Vector Magnetograph (Grigoryev et al., 1985), and a magnetograph at Huairou Solar Observing Station (HSOS, Ai \& Hu, 1986). For additional review, see Jones (1993). Later developments include the Imaging Vector Magnetograph (IVM, Mickey et al., 1996), the Diffraction Limited Spectropolarimeter (DLSP, Sankarasubramanian et al., 2006), the Zürich Imaging Polarimeters (ZIMPOL I and II, Povel, 2001), the La Palma Stokes Polarimeter and Tenerife Infrared Polarimeter (LPSP and TIP, Mártinez Pillet et al., 1999), and the HINODE Spectro-Polarimeter (SP, Lites et al., 2013). All of these instruments had a limited field of view, typically about the size of an average active region. Full disk vector magnetograms have been routinely observed since late 2003 by VSM/SOLIS (Keller et al., 2003). Beginning in 2010, full disk vector magnetograms have been available from the Helioseismic and Magnetic Imager (HMI, Scherrer et al., 2012) on board the solar dynamics observatory (SDO).

The HMI instrument is a filtergraph covering the full solar disk with $4096 \times 4096$ pixels. The spatial resolution is about $1^{\prime \prime}$ with a $0.5^{\prime \prime}$ pixel size. The width of the filter profiles is $76 \mathrm{~m} \AA$. The spectral line used is Fe i $6173 \AA$, which forms in the photosphere (Norton et al., 2006). The Stokes parameters $(I, Q, U, V)$ are computed from those measurements (Couvidat et al., 2016), and are further inverted to retrieve the vector 
magnetic field using a Milne-Eddington (ME) based inversion algorithm, the Very Fast Inversion of the Stokes Vector (VFISV Borrero et al., 2011; Centeno et al., 2014). To suppress $p$-mode oscillations and to increase the signal-to-noise ratio, registered filtergrams are averaged over a certain time before computing the Stokes vector. By default a weighted average is computed every $720 \mathrm{~s}$ using data obtained over $1350 \mathrm{~s}$; other averaging windows are also available.

Inversion of the vector field has an unavoidable $180^{\circ}$ ambiguity in the azimuthal field direction. Assumptions about the field must be made to resolve this ambiguity. For all pixels in active regions, as well as for strong-field pixels (where the $S / N>3$ in the transverse signal plus a $50 \mathrm{G}$ buffer) on the quiet Sun regions, the azimuth is determined using a minimum energy algorithm (Metcalf, 1994; Metcalf et al., 2006; Leka et al., 2009; Hoeksema et al., 2014). The minimum-energymethod computation is time consuming for pixels where the signal is dominated by noise. So for weaker polarization regions the $180^{\circ}$ ambiguity is solved using three quicker methods: a randomizing method (the option to add $180^{\circ}$ is determined randomly), an acute-angle comparison to a potential field, and a method that provides the most radially-directed solution. More details can be found in Hoeksema et al. (2014).

The VSM is a spectrograph-based instrument which observes full line profiles of the Fe i 6301.5 and $6302.5 \AA$ spectral lines with spectral sampling of $2.4 \mathrm{pm}$ and pixel size of $1.0 \times 1.0(1.14 \times 1.14$ before January 2010) arcseconds over a $2048 \times 2048$ pixel field of view. To construct a full-disk magnetogram, the image of the Sun is scanned in the direction perpendicular to the spectrograph slit. At each scanning step, the spectra of each pixel along the slit is recorded simultaneously. With each scanning step taking $0.6 \mathrm{~s}$ a full disk magnetogram can be completed in about $20 \mathrm{~min}$. Since the spectrograph slit is curved, geometric distortions are introduced to the image of the Sun. These distortions are corrected by shifting the position of each pixel in the final image to the closest integer position of the true pixel location on the round-Sun image. The maximum uncertainty in the position of a pixel does not exceed half-apixel thus is significantly smaller than the typical atmospheric seeing for this groundbased instrument. The above correction procedure avoids ill-posed interpolation of full disk magnetograms and preserves the mapping of spectral information for each image pixel.

Similar to HMI, the observed Stokes profile parameters $Q, U$, $V$, and $I$ are inverted using the VFISV code under the assumption of a standard Milne-Eddington stellar atmosphere. However, unlike HMI, the VSM inversion includes the magnetic-field filling factor $(\alpha)$ as an additional fit parameter, which represents the fraction of each instrument pixel filled by magnetized plasma. For additional details about SOLIS/VSM inversion methods and pipeline, see Harker (2017). The $180^{\circ}$ azimuthal ambiguity in the transverse field is resolved using the Very Fast Disambiguation Method (VFDM, Rudenko \& Anfinogentov, 2014). The VFDM has an accuracy almost as good as that of the minimum energy method (used for HMI disambiguation) but is much faster. For a synoptic instrument such as VSM and HMI, the disambiguation is done automatically as part of the pipeline data reduction. The pipeline reductions are optimized for "a good answer most of the time, in time for the next dataset" but in some cases may not return the best possible solution.

\section{Notes on digitization of historical measurements of magnetic field}

Recently, there has been a strong resurgence in the use of historical observations of the Sun's magnetic field. For example, these data have been employed in the study of long-term trends in sunspot field strengths (e.g. Livingston et al., 2006; Penn \& Livingston, 2006; Pevtsov et al., 2011, 2014b; Watson et al., 2011; Rezaei et al., 2012) and sunspot-area - maximum field strength relation (Nagovitsyn et al., 2017). The data have also been implemented to the study the tilt of active regions (Joy's law Tlatova et al., 2018), reconstruction of polar fields (Pevtsov et al., 2016) and magnetic momentum of active regions (Virtanen et al., 2019b).

Historical records of sunspot magnetic field measurements from MWO for 1917-2016 were digitized (Pevtsov et al., 2019b). 1957-1997 observations from Pulkovo, CrAO, and other observatories from Russia and other former Soviet Union republics have also been digitized (for details, see Pevtsov et al., 2011). Observations from CrAO (1998-present) and Potsdam/ AIP have not been fully digitized yet.

The photographs of the oscillograms from the earliest Babcock magnetograph and the original pen-plot magnetograms are stored in the archive of the Carnegie Observatories in Pasadena, California. While these images could be converted to a digital format, their interpretation would be quite challenging. For example, the images of oscillograms are distorted due to the way the cathode ray tube (CRT) display was photographed onto $35 \mathrm{~mm}$ film. There are likely to be many calibration issues including the zero level. In respect to the zero point offset, Jack Harvey recalls that when he observed with the second and third generation magnetograph, "setting the zero was rather $a d$ hoc (turn off the modulator) and [the] calibration was also rather rough (involving shims and a meter stick at first). The selsyn servo system that scanned the image would frequently slip and distort the image. [...] the electro-mechanical relays were used to define the transition from $1 \mathrm{G}$ threshold to another and tweaking the little adjustment screws was finicky. These relays were not in the first generation system so it might actually be better calibrated."

The scientific usefulness of these early magnetograms maybe limited. The 1952-1954 magnetograms were carefully analyzed by Babcock \& Babcock (1955), and Babcock (1959) did the analysis of the 1956-1959 polar observations. It is not clear how much more could be obtained from these early data. The Carnegie Observatories archive also contains a sizable collection of the pen-plot magnetograms. As the examples in Figures 4 and 5 show, the automatic conversion of these data to digital magnetograms could be challenging. Moreover, the colors used in these magnetograms maybe fading. The colors are critical for distinguishing the polarity of the magnetic fields. These aspects may need to be taken into consideration when making a decision about the digitization of these early magnetograms.

\section{Magnetogram cross-calibration}

The magnetic fields are derived using instruments, which employ different observational techniques (e.g., spectropolarimeters vs. imaging magnetographs, Sect. 4). The instruments 

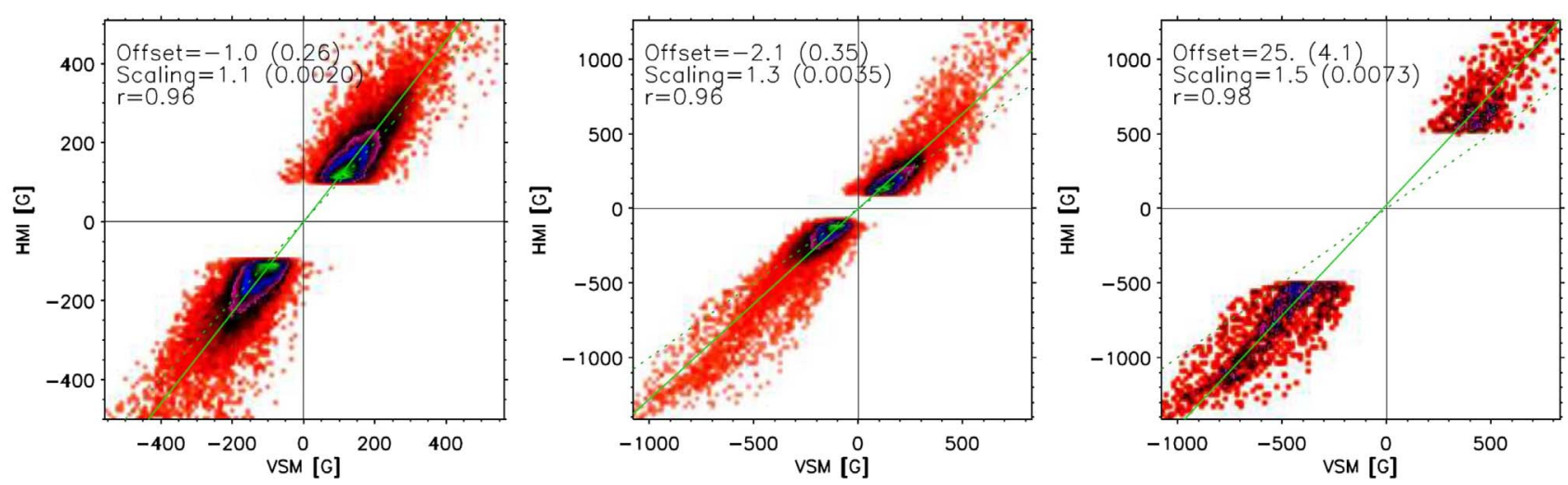

Fig. 7. Pixel-by-pixel comparison of VSM and HMI longitudinal magnetograms observed on 14 November 2011. The HMI magnetogram was spatially smoothed to match VSM resolution. Three panels show scatter plots for various flux ranges (color scale for scatter plots is logarithmic). Shown in solid line is computed fit and in dotted line scaling factor equal unity and zero offset fit. Computed fit (scaling factor and offset, fit standard deviation in parentheses) and Pearson correlation coefficient are given in upper left corner of each panel. Reproduced from Pietarila et al. (2013).

may have quite different spatial and spectral resolution, and they may employ different techniques for deriving the magnetic field from the observations (e.g., full Stokes inversions or simplified methods such as center-of-gravity). There could also be differences in correcting the instrumental polarization etc. Thus, it should not be a surprise that the observations taken by different instruments may differ in the amplitude and direction of the magnetic fields. The comparison and cross-calibration of magnetograms from different instruments was a part of the field from its very beginning (e.g., Makita et al., 1985b; Hofmann et al., 1988a, b; Ronan et al., 1992; Jones \& Ceja, 2001; Berger \& Lites, 2002, 2003; Hagino et al., 2009; Liu et al., 2012; Bai et al., 2014; Riley et al., 2014; Sainz Dalda, 2017). In respect to the two major full disk instruments, Pietarila et al. (2013) found that HMI/SDO and VSM/SOLIS have a scaling coefficient close to unity if the higher resolution (HMI) data are smoothed to match the lower resolution (VSM) magnetograms. Figure 7 shows a pixel-by-pixel scatter plot comparisons of a longitudinal magnetogram taken on 14 November 2011. The first degree polynomial fit to the scatter plots indicates a change in the scaling factor from 1.1 for the magnetic flux in the range $\pm 400 \mathrm{G}$, to about 1.5 for stronger field $(500-2000 \mathrm{G})$, see Pietarila et al. (2013). The scaling factors were different for different locations of the solar disk. Riley et al. (2014) compared the Carrington rotation maps (see Sect. 7) from seven instruments (i.e. WSO, KPVT, SOLIS, GONG, MDI, MWO, and HMI) and found that the scaling coefficients vary with the sine of latitude. Furthermore, the scaling coefficients were different for different synoptic maps, although the authors concluded that the long-term variations in the scaling coefficients were not significant. Since the scaling may be non-linear with different scaling coefficients for weaker and stronger fields, Virtanen \& Mursula (2017) suggested scaling the magnetograms from different instruments by scaling the different spherical harmonics derived from the corresponding data. As a more recent development, Plowman \& Berger (2020) described a magnetograph calibration based on artificial data, where the output of a solar atmosphere model (with the magnetic field) is used to synthesize the solar spectral line profiles. The line profiles are then ran through an instrument simulator, whose parameters match the magnetograph under the investigation, and the derived magnetic fields are compared with the known magnetic fields in the initial model. If successful, this approach would enable a much better calibration of the magnetograph outputs. Similar instrument simulator was developed early by Blanco Rodríguez et al. (2018).

\section{Carrington rotation synoptic charts}

Carrington rotation synoptic charts are used widely to better represent the solar activity and its changes at every location on the solar "surface". Such synoptic charts are constructed over a full solar rotation (about 27 days), by adding new observations of a visible portion of solar disk to the observations taken in early periods (e.g., Ulrich \& Boyden, 2006, and references therein). The coordinate system (latitude-longitude) in these maps is based on 27.2753 days synodic rotation rate, with the first rotation starting November 9, 1853 as defined by Carrington (1863). More recent example of modern day Carrington rotation synoptic maps can be accessed via the databases of the individual instruments, for example, GONG (https://gong2.nso.edu/archive/ patch.pl?menutype=f), VSM/SOLIS (https://solis.nso.edu/0/ vsm/vsm_maps.php), MDI/SOHO, and HMI/SDO (http://hmi. stanford.edu/data/synoptic.html). Figure 8 shows one of the first magnetic field Carrington synoptic charts. The maps are created using different algorithms. For example, VSM/SOLIS use the full disk observations weighted by the distance from the disk center (e.g., Worden \& Harvey, 2000; Ulrich \& Boyden, 2006; Bertello et al., 2014, and the references therein), while the HMI/SDO maps only use a narrow longitudinal strip of data near the central meridian (see http://jsoc.stanford.edu/HMI/ LOS_Synoptic_charts.html). Since then, such maps have become a "workhorse" for studying the evolution of large-scale magnetic fields, solar corona and solar wind modeling, and in operational space weather forecasting. Until recently, synoptic maps were created under a restrictive assumption that the magnetic field is radial to the surface of the Sun. Then the pseudo-radial component was derived from the line-of-sight 


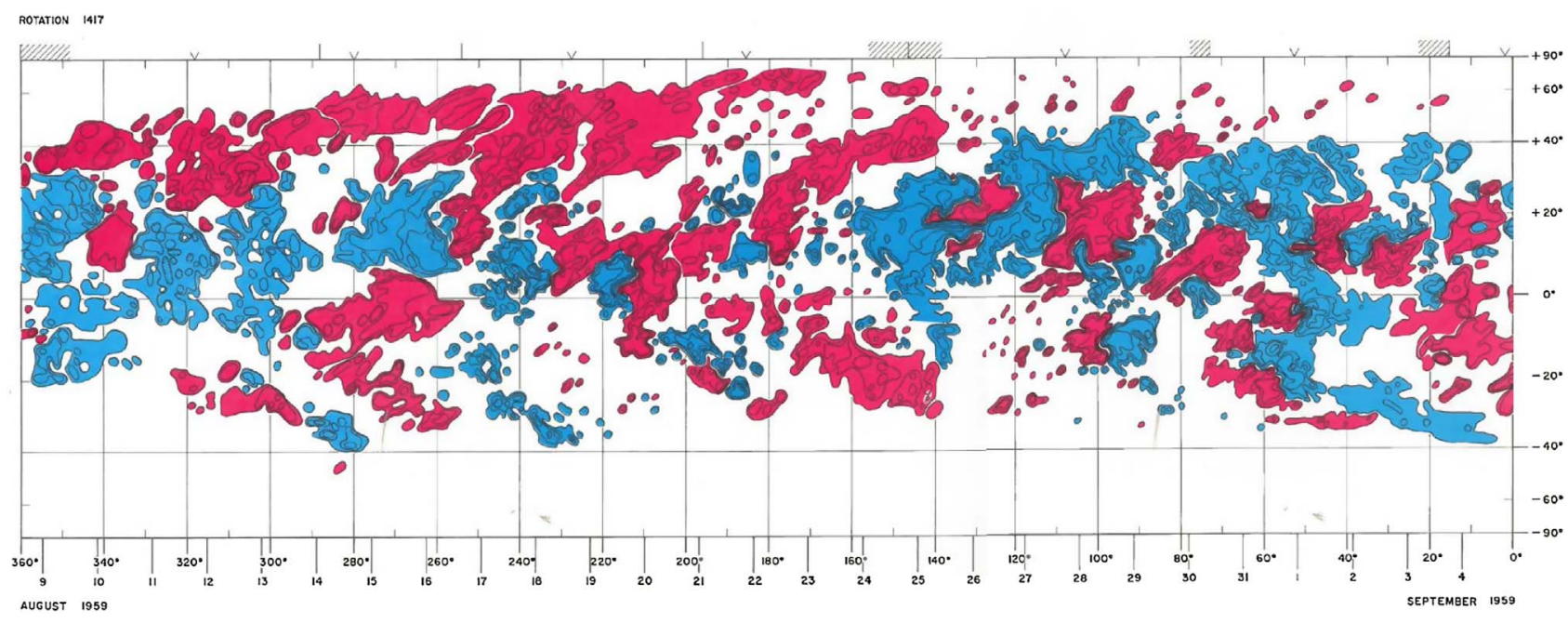

Fig. 8. Example of one of the earliest Carrington rotation (CR1417) synoptic maps of magnetic field (August 1959) from Howard et al. (1967). Blue/red corresponds to negative/positive polarity magnetic field. Due to a large size of the printouts, it required two separate scans to cover a single map. Two images are joint at about $130^{\circ}$ longitude.

$B_{\text {long }}$ magnetograms divided by the cosine of a viewing angle. With regular observations of the full disk vector magnetic field, vector field synoptic maps have been recently constructed (Gosain et al., 2013; Virtanen et al., 2019a).

Observations of magnetic fields provide key information for developing our understanding of the Sun's short-term (space weather) and long-term (space climate) activity and in predicting these effects on the Earth.

Synoptic full disk longitudinal magnetograms have exist since the late 1960s. These data continue to serve as the primary input for the space weather and space climate research and operational forecasts. By their nature, longitudinal magnetograms do not contain sufficient information to derive the true orientation of the magnetic-field vectors, and thus, require additional assumptions for physical interpretation. For example, "pseudo-radial field" synoptic maps, which are widely used in space weather forecasting are created under the assumption that the true field is radial.

In principle observations of vector Stokes polarimetery have the necessary information to fully reconstruct photospheric vector-magnetic-field maps. Efforts are being made to start employing such data in operational space weather forecasts.

The individual Carrington rotation synoptic maps provide a good representation of the evolution of individual features over the course of several rotation. They allow the identification of the recurrent active regions (those that lasted longer than one solar rotation), as well as the clustering of new active regions in so called "complexes of activity", "active longitudes", etc (e.g., Bumba \& Howard, 1969; Brouwer \& Zwaan, 1990; van Driel-Gesztelyi \& Green, 2015; Gyenge et al., 2016). Averaging the synoptic maps in longitudinal direction, and putting them next to each other over the period of many solar rotations (one or several solar cycles) will form a "supersynoptic map" (Ulrich, 2001) or a "magnetic butterfly diagram" (e.g., Fig. 17 in Hathaway, 2015).

The super-synoptic map (see Fig. 9) exhibits several important properties that describe the long-term evolution of magnetic fields. First, the sunspot activity cycle starts with active regions emerging at mid-latitudes in each hemisphere. Then as the cycle progresses, the latitude of new sunspot emergence gradually drifts towards the equator. This latitudinal drift forms a characteristic pattern resembling a pair of butterfly wings, which explains the name of the "butterfly diagram" (Maunder, 1904). Because of the asymmetry in properties of the leading and following polarities within a sunspot group, i.e. the sunspots of the leading polarity are situated closer to equator (see Sect. 9), they tend to be more compact and have a stronger field strength (see Fig. 5 in Tlatov et al., 2015; Vitinsky et al., 1986; Fan et al., 1993; Fisher et al., 2000, and references therein), supersynoptic maps exhibits a slight asymmetry in the average polarity of sunspot groups in each hemisphere (according to the Hale polarity rule). In addition, the map shows a much weaker patterns of the magnetic flux transported from the decaying active regions to the solar poles. These poleward surges originate mostly from the decaying flux of the trailing polarity, and play a major role in cancelling out the polar fields of a solar cycle, and build up of the polar field for the next cycle sunspot (e.g., Hathaway \& Upton, 2014, also see Sect. 8 for discussion of the evolution of polar magnetic flux in the framework of surface flux transport dynamo). Figure 9 exhibits early indications of the up incoming cycle 25 . Due to a significant longitudinal averaging, a contribution of a small single sunspot group may not be obvious in the supersynoptic map. Nevertheless, sunspot groups whose polarity orientation and latitude correspond to cycle 25 have already been observed in the locations marked by two black arrows in Figure 9. Similar indicators were also observed in the very early phase of cycle 24 . Based on the latitude of these early sunspots (slightly higher than the latitude of sunspots at the beginning of cycle 24), and the strength of the polar field (about the same as at the minimum of cycle 23, Fig. 10), we expect that the amplitude of cycle 25 will be similar (or maybe even slightly higher) as of the cycle 24 . Our prediction is based on two statistical tendencies about the latitude of emergence of sunspots at the beginning of cycle and the amplitude of cycle (Solanki et al., 2008; Tlatov \& Pevtsov, 2010) and the dependence of amplitude of solar cycle on the 


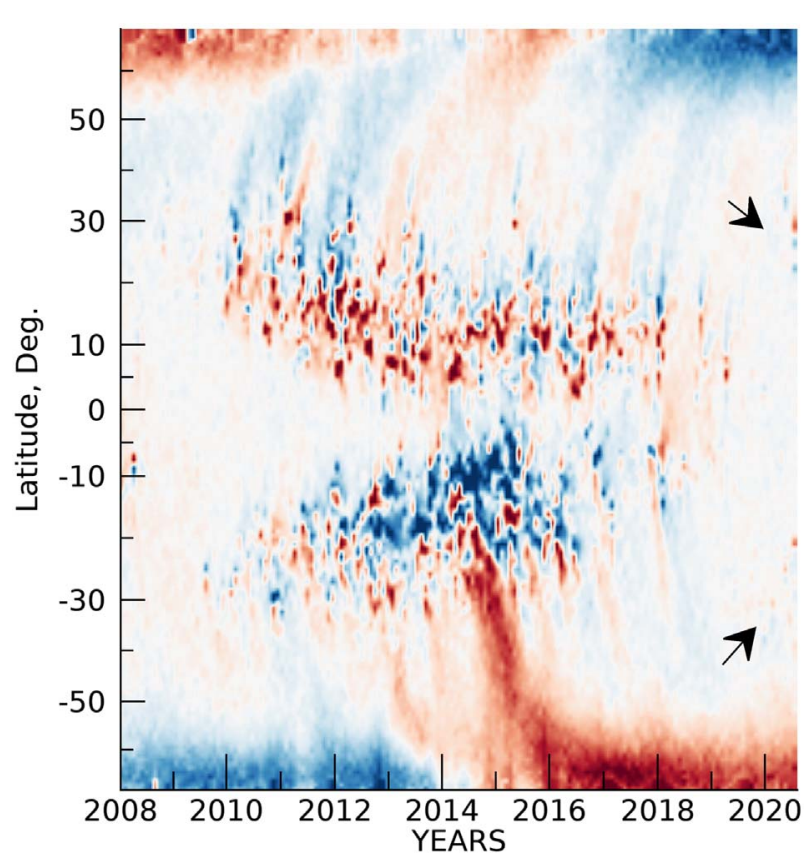

Fig. 9. Supersynoptic map for Cycle 24 based on the (zero-point corrected) integer rotation synoptic maps from GONG. Blue/red show negative/positive polarity fields scaled between $\pm 5 \mathrm{G}$. Two black arrows mark approximate location of two latitudinal bands of Cycle 25.

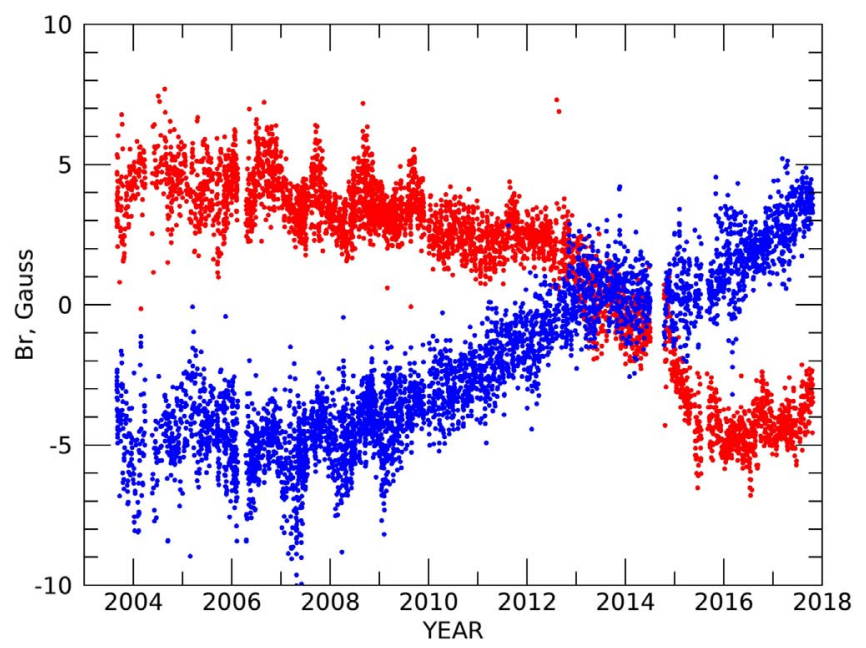

Fig. 10. Average magnetic flux density (a) between $60^{\circ} \mathrm{N}-75^{\circ} \mathrm{N}$ (red) and $-60^{\circ} \mathrm{S}-75^{\circ} \mathrm{S}$ (blue) derived using longitudinal magnetograms from VSM/SOLIS under the assumption that the magnetic field is radial.

amplitude of polar field during the minimum of sunspot cycle (Petrovay, 2020).

\section{Polar fields}

The earliest (unsuccessful) attempts to measure the global magnetic field of the Sun including polar areas were made in
1908 and then again in 1911 by Hale (1912). In the follow up studies, Hale and his co-workers (Hale, 1913; Hale et al., 1918) conducted a detailed examination of additional measurements, and found the presence of a dipolar field. The validity of these early findings was questioned by later researchers (see, Stenflo, 2017, and references therein), who rightfully pointed out that the amplitude of the polar fields reported in these early articles was too high. In fact, Stenflo (1970) remeasured the original photographic plates used in Hale et al. (1918) using a scanning microdensitometer, and did not confirm the hemispheric trend in global field reported by Hale and his co-workers. The first magnetograph measurements provided clear evidence of polar magnetic fields (Babcock \& Babcock, 1955) and their reversals (Babcock, 1959), see Figure 9. These observations were put into perspective of the 22-year magnetic cycle of solar activity (Babcock, 1961), first noted by Hale \& Nicholson (1925, see also Cliver, 2014).

According to a semi-empirical Ballcock-Leighton model (Babcock, 1961; Leighton, 1969), at the beginning of each cycle, the poloidal field (represented by the polar magnetic field) serves as a seed field for the production of (toroidal) magnetic field of active regions. From this, it follows that the strength of the polar field during the minimum of sunspot activity could serve as a good predictor of the strength (amplitude) of the next solar cycle (Schatten et al., 1978; Petrovay, 2020, and references therein). Figure 10 shows the mean flux in polar areas (between $60^{\circ}$ and $75^{\circ}$ latitude in the Northern and Southern hemispheres derived from the longitudinal magnetogram observations from VSM/SOLIS. The strongest polar fields were observed during 2006-2007, and the polar field reversals occurred over the extended period during 2013-2014. For these calculations, the magnetic field at/near the poles was assumed to be radial, and thus, the correction is made by dividing the observed line-ofsight component by a cosine of the viewing angle. Still, the pseudo-radial field computed this way shows clear annual variations, thus, suggesting that the field is not radial. While some previous studies have suggested that the polar fields are "nearly" radial (e.g., Svalgaard et al., 1978; Petrie, 2017), Ulrich \& Boyden (2006) found a slight poleward inclination of the magnetic field, while Virtanen et al. (2019a) described an equator-ward inclination. To arrive at these conclusions, Ulrich \& Boyden (2006) found an inclination angle by minimizing the amplitude of annual variations in high-latitude fields (as in Fig. 10), while Virtanen et al. (2019a) used the vector field observations from VSM/SOLIS. Applying the approach used by Ulrich \& Boyden (2006) to the data shown in Figure 10, we found that the amplitude of the annual variations between 2006 and 2009 could be reduced if the field in the latitudinal range of $60^{\circ}-75^{\circ}$ was vertically inclined in both hemispheres by about $3^{\circ}$ in the direction of the equator. In 2004-2005 and 2010-2017 the magnetic field appears to have a much smaller inclination if any. This indicates that questions about the radiality of the photospheric magnetic fields at high latitudes require further investigation.

Figure 11 provides an example of the evolution of the polar field at the Southern solar pole from the declining phase of cycle 23, the polar field reversal in 2013-2014, and the formation of a new polar field in early 2017. By early 2004 (see Figs. 10 and 11a), the southern pole showed a well-defined polar field cap of the negative polarity (Fig. 11a). At that time, the negative polarity cap was already completely surrounded by the opposite 


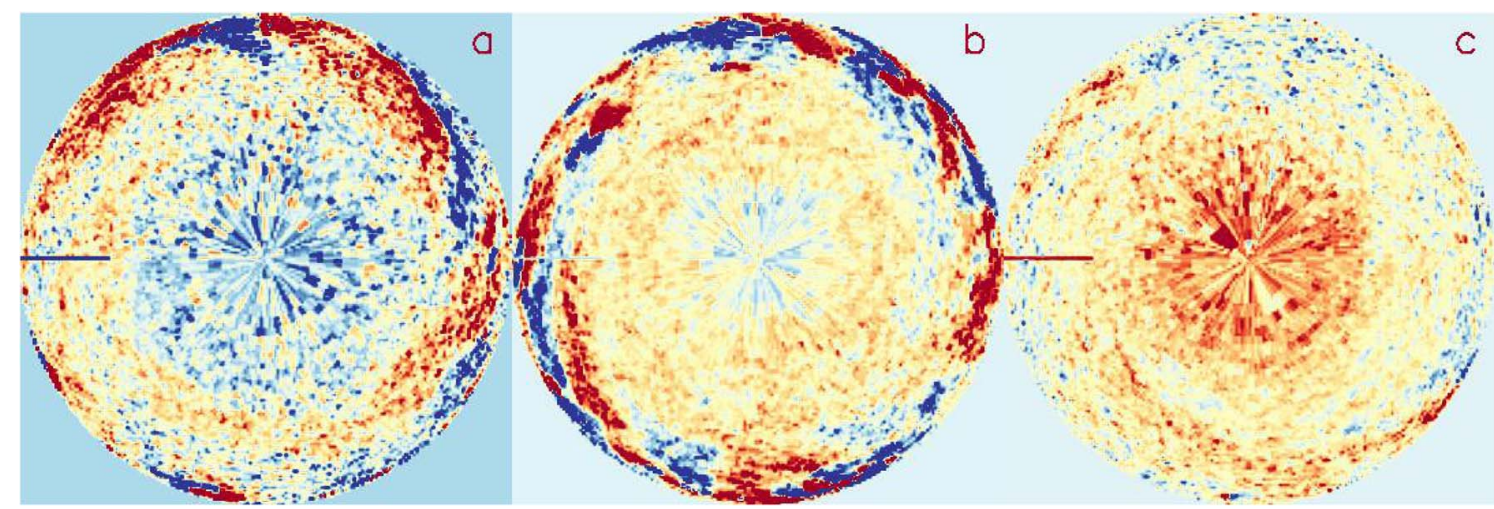

Fig. 11. Polar view of the Southern Pole during (a) January-February 2004, CR2012, (b) January-February 2013, CR2133, and (c) January 2017, CR2186 using the synoptic maps of pseudo-radial magnetic field from VSM/SOLIS. Positive/negative polarities are shown as reddish/ bluish halftones. The magnetograms are scaled between $\pm 15 \mathrm{G}$. Horizontal line in panels (a) and (c) corresponds to the beginning and end of the Carrington rotation. In this projection, the solar (Carrington) longitudes start at the horizontal line at $360^{\circ}$ and decrease in the clockwise direction to $0^{\circ}$ back at the horizontal line. The solar rotation as viewed from Southern pole is in the counterclockwise direction. Since these images are constructed from the Carrington rotation maps with the mean solar rotation removed, only the component related to the differential rotation is present in these images. For more detailed picture of the evolution of polar field see the accompanying video.

(positive) polarity field indicating that the process of cancelling out of the polar field had already begun. The poleward surges, which in this projection appear as counterclockwise spirals started in low latitudes and ended in high latitudes (closer to the disk center in this projection, see Figs. 11a and 11c). At the beginning of the polar field reversal (January-February 2013, CR2133), the negative polarity was significantly weakened. It was surrounded by a broadly distributed weak positive polarity, which represented the future polar field (Fig. 11b). This diffuse polarity had concentrated into a smaller polar cap by early 2017 (Fig. 11c). The Southern pole field reversal can be seen in more detail in the accompanied movie (Supplementary Movie 1), which uses 2-3 Carrington rotations around December-March each year from SOLIS observations, when the Southern pole enjoys better visibility from Earth. The radially looking pattern appearing near the solar disk center (Southern pole) in Figure 11 is a combination of the poor sampling of polar regions from the ecliptic plane (a single pixel in the image plane may cover an extended range of latitudes near the solar limb as observed from Earth), and the patchy structure of the polar fields as discovered by the observations from HINODE (Tsuneta et al., 2008; Petrie, 2017). Future investigations would need to overcome such limitations, perhaps by using observations taken from outside the ecliptic plane.

\section{East-west orientation of active regions}

Sunspots develop in the photosphere in groups (active regions), with the preceding and the following spots having opposite magnetic polarity. Furthermore, the leading polarity of active regions in the Northern hemisphere is opposite in sign to the leading polarity of groups in the Southern hemisphere, and the sign of the leading polarity in each hemisphere reverses from one sunspot cycle to the next. This polarity orientation is called the Hale polarity rule. The leading sunspot of the group tends to be situated at a lower latitude (closer to the equator) as compared with the following polarity sunspot. If one draws an imaginary line connecting the preceding and trailing sunspots of a group, the line would appear tilted relative to direction parallel to the solar equator. The latitudinal variation of tilt angle is now called the Joy's law (as introduced by Zirin, 1988). Both tendencies were first described by Hale and his co-workers (Hale et al., 1919; Hale \& Nicholson, 1925), and had been the subject of numerous studies since then (for review, see Pevtsov et al., 2014a, and references therein). The orientation of sunspot groups can be explained in the framework of the Babcock-Leighton model (Babcock, 1961; Norton \& Gilman, 2005; Kosovichev $\&$ Stenflo, 2008), in which the active regions represent the upper (photospheric) portion of the toroidal flux tubes. Several properties of Joy's law have being successfully explained by the effects of the Coriolis force on a flux tube rising through the convection zone (for review, see Fan et al., 1993; Fisher et al., 1995, 2000; Pevtsov et al., 2014a, and references therein). Other explanations include the kink-instability in the photospheric portion of the flux tube forming the active region (Leighton, 1969; Longcope et al., 1999; Holder et al., 2004).

The orientation of active regions may affect the transport of the magnetic flux of decaying active regions. For example, larger tilts will enhance the poleward transport of the magnetic flux of trailing polarity, and depending on the polarity orientation, could either strengthen or weaken the polar field (e.g., Baumann et al., 2004; Virtanen et al., 2017; Whitbread et al., 2017; Petrovay \& Talafha, 2019). Several studies of the Hale polarity rule and the tilt angle of active regions were published in recent years (Illarionov et al., 2015; Li, 2018; Tlatova et al., 2018; Kuzanyan et al., 2019; Kleeorin et al., 2020; Poisson et al., 2020). Thus, for example, Poisson et al. (2020) emphasized the importance of, so called, magnetic tongues in the determination of active region tilts using longitudinal magnetograms. The magnetic tongues, or elongations of two main polarities of an active regions during their emergence were first reported by López Fuentes et al. (2000). Poisson et al. (2020) have shown that the active regions with weaker magnetic tongues show the magnetic tilts similar to those derived from white light 


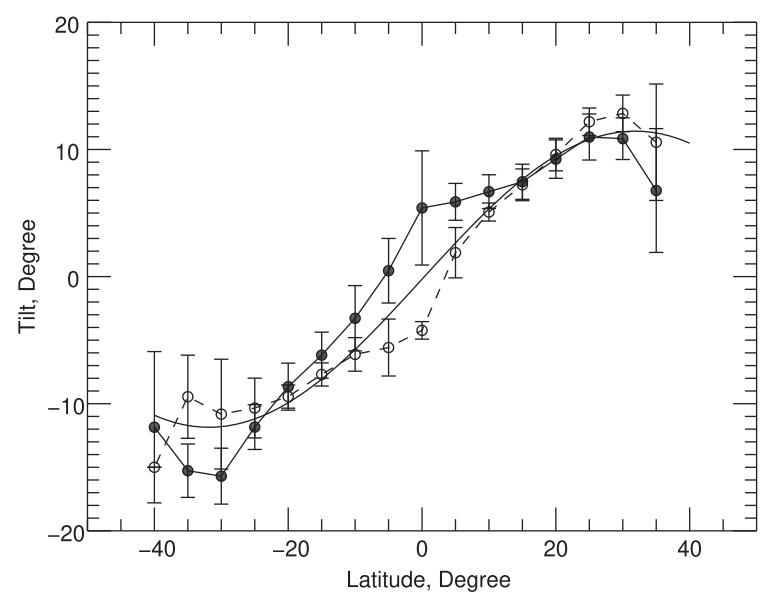

Fig. 12. Mean tilts of sunspot groups for odd (filled circles, dotted line) and even (open circles, dashed line) cycles. Solid line represents fit to all dataset, $\gamma=(0.2 \pm 0.08) \sin (2.80 \varphi)-(0.00 \pm 0.06)$, where $\varphi$ is the latitude. Error bars correspond to one sigma standard deviation of mean. Reproduced by permission from Tlatova et al. (2018).

images. However, for active regions with strong magnetic tongues, there could be significant difference in tilts derived from white light images and magnetograms.

Traditionally, the latitudinal dependence of tilt angles was represented by a monotonic function of latitude or its sine. Some recent studies, however, question this monotonic behaviour. Figure 12 shows the latitudinal dependence of active region tilts separately for odd- and even- numbered solar cycles as derived by Tlatova et al. (2018) using MWO sunspot drawings. The plot shows two distinct features: a non-monotonic behaviour of tilts at higher latitudes (a decrease in tilt angles), and non-zero tilts at the equator (positive tilt for odd-numbered cycles and negative tilt for even cycles). The non-monotonic behavior in tilt angle with latitudes can be explained by the changes in magnetic properties of active regions with the phase of solar cycle. The regions emerging at high latitudes at the beginning of solar cycle tend to be smaller in size and have weaker magnetic flux. Those developing around the sunspots' maximum tend to be larger in size and stronger in its magnetic flux. Since the action of the Coriolis force on emerging flux tube depends on their size and the flux (Fisher et al., 1995), it could explain the presence of a maximum in the latitude-tilt dependence as found by Tlatova et al. (2018). The non-zero tilts at the equator could be explained by a displacement of the magnetic equator relative to the rotational equator. Recently, Kuzanyan et al. (2019) and Kleeorin et al. (2020) proposed an alternative explanation; that the active region tilt could be a joint action of the Coriolis force and magnetic tension (Ampére's force). At high latitudes, the tilt will be determined mostly by the Coriolis force action. However, in low latitudes, the magnetic contribution could be significant. The sign of this contribution depends on the polarity of polar field, and thus, could result in the non-zero tilt of opposite sign for odd- and even-numbered cycles.

The Joy's law and the Hale polarity rule represent different aspects of the same phenomenon of the orientation of active regions in the photosphere. The active region tilts show significant scatter relative to a mean value for each latitude. By contrast, the polarity orientation (the Hale polarity rule) is a strong dependence. Past studies have found that the fraction of active regions (annual or total over the studied dataset), which deviate from the rule (called non-Hale or anti-Hale regions) are usually smaller than $10 \%$ of all active regions: 1.4-6.3\% (Hale et al., 1919, MWO sunspot 1913-1917 dataset), $<4 \%$ (Stenflo \& Kosovichev, 2012, MDI/SOHO), and between $6.5 \%$ and $9.1 \%$ (Li \& Ulrich, 2012, Cycles 21-23), and $(8.1 \pm 0.4) \% \mathrm{Li}(2018$, MDI and HMI data). McClintock et al. (2014) reported a larger fraction of anti-Hale regions in Cycles 20 and 24. However, these two cycles were only partially covered by available data. McClintock et al. (2014) also reported that the fraction of anti-Hale regions increase during the minimum of sunspot cycle. Mordvinov et al. (2016) also observed a significant number of small active regions of non-Hale polarity orientation in low latitudes during the minimum between cycles 23 and 24 . The poleward transport of the magnetic flux of these non-Hale regions may explain the evolution of polar fields between 2013 and 2015 (multiple reversals and slow polar field rebuild, see Sect. 8 and Fig. 10).

According to Li (2018), Hale and non-Hale active regions show similar latitudinal distributions, and follow a similar Joy's law in their tilts. However, Illarionov et al. (2015) showed that small magnetic bipoles form a separate subset, with their polarity orientation has being opposite in sign to those of regular sized active regions.

\section{Magnetic flux vs. area}

In the framework of a simple magnetic flux tube, which is in a hydrostatic equilibrium with its surroundings, the outside gas pressure will be balanced by the sum of the internal gas and the magnetic pressure. The latter is proportional to the magnetic field strength squared. Assuming that the external gas pressure in the photosphere is the same for all sunspots, the size (diameter or area) of a sunspot could serve as a proxy for the magnetic flux inside the sunspot or for its maximum field strength. This simple approach enables deriving some information about the magnetic properties of sunspots, well past the earliest observations of magnetic fields in sunspots.

The relationship between the magnetic field strength $\mathrm{H}$ in a sunspots umbrae and its area $\mathrm{S}$ can be represented by several functional forms (Nicholson, 1933; Houtgast \& van Sluiters, 1948; Ringnes \& Jensen, 1960; Ringnes, 1965; Pevtsov et al., 2014b; Muñoz-Jaramillo et al., 2015; Nagovitsyn et al., 2017):

$$
\begin{gathered}
H=A+B \times \log S \\
\log H=A_{1}+B_{1} \times \log S \\
H=\frac{A_{2} \times S}{B_{2}+S} \\
H=a \times S^{b},
\end{gathered}
$$

where $A, B, A_{1}, B_{1}, A_{2}, B_{2}, a$, and $b$ are scaling coefficients found by fitting. Using a known magnetic field distribution along with the radial distance inside a sunspot, one can also 

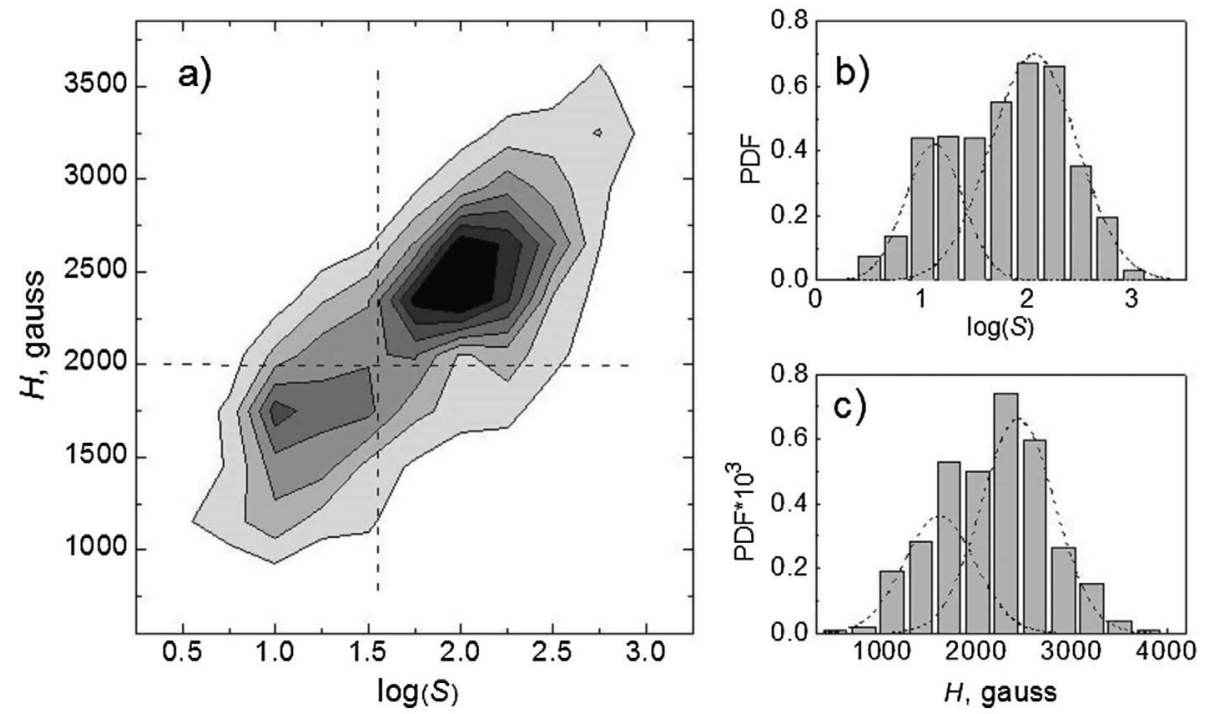

Fig. 13. Probability distribution functions (PDF) for (a) umbral field strength and sunspot area, (b) sunspot area and (c) umbral field strength. Dashed lines in panels (b) and (c) delineate two components contributing to each histogram. Vertical and horizontal dashed lines in panel (a) indicate approximate division between two components (small/weak and large/strong sunspots). Overall trend in $H$ vs. $\log S$ (panel a) agrees with equation (1). Reproduced from Nagovitsyn et al. (2017).

estimate the total magnetic flux $\Phi$ in sunspot (e.g., Nagovitsyn et al., 2016):

$$
\Phi=c H \cdot S,
$$

where $c$ is a coefficient determined by a specific magnetic field distribution inside a sunspot. The relation between the sunspot (or umbral) area and the maximum field strength, $B_{\max }$ in the near infrared (Si I 10827.09, Fe I 10896.30 and Fe I $15648.52 \AA$ spectral lines) has been studied by Rezaei et al. (2012). They found a statistically significant correlation between the total area of a sunspot $S$ and $B_{\max }$ and a linear relation $B_{\max } \propto 0.93 S$, where $B_{\max }$ is in units of Gauss and area is in $\operatorname{arcsec}^{2}$. A similar correlation for umbral areas is much weaker. Sunspots with the rudimentary penumbra may significantly deviate from $S$ to $B_{\max }$ dependency (see, Fig. 5 in Rezaei et al., 2012).

All functional dependencies (Eqs. (1)-(4)) show a good correlation between the magnetic field $H$ and sunspot area $S$ albeit with a significant scatter. On the basis of a statistical analysis Ringnes \& Jensen (1960) concluded that equation (1) provides the best representation of $\mathrm{H}$ vs. $\mathrm{S}$ dependence. Figure 13a, which is plotted in $H$ vs. $\log S$ coordinates seem to support their conclusion. On the other hand, Tlatov \& Pevtsov (2014) concluded that both equations (1) and (2) work equally well. Assuming that the physical relation between $H$ and $S$ is due to the action of the magnetic pressure in sunspots, equation (2) is more suitable to represent such a relation. Also, equation (2) can be derived from the magnetic field distribution of a dipole situated at a certain depth below the photosphere (Ikhsanov, 1968; Tlatov \& Pevtsov, 2014).

Coefficients $A_{1}$ and $B_{1}$ were found to show long-term (longer than a solar cycle) variations (Ringnes, 1965; Pevtsov et al., 2014b; Nagovitsyn et al., 2017). Pevtsov et al. (2014b) found a correlation between the $B_{1}$ coefficient and the amplitude of a solar cycle. They related this to the changes in the relative fraction of small and large sunspots in each cycle (i.e., larger the fraction of large sunspots in selected solar cycle - steeper the $H$ vs. $S$ dependence for this cycle).

Figure 13a shows a $2 \mathrm{D}$ probability distribution function (PDF) of sunspots field strength and sunspot area for 653 sunspots observed during 1994-2013. Sunspot areas are taken from the observations at KMAS, and sunspot field strengths are from the measurements at CrAO. The relation between sunspot area and the maximum field strength in sunspots is quite clear. Nagovitsyn et al. (2017) argued that the 2D PDF shows two distinct peaks representing a bimodal distribution of sunspot areas and their magnetic fields. One component of the distribution corresponds to small sunspots and the other to large sunspots. The presence of such a bimodal distribution was confirmed by several authors (Kuklin, 1973, 1980; Nagovitsyn et al., 2012, 2018; Cho et al., 2015; Muñoz-Jaramillo et al., 2015; McClintock \& Norton, 2016; Nagovitsyn \& Pevtsov, 2016; Kostyuchenko, 2017; Tlatov et al., 2019, and references therein).

Nagovitsyn et al. (2012) suggested that the two populations could represent the action of two spatially separated dynamos: one operating at the base/bulk of the convection zone, and the other - near the surface (see also, Clette \& Lefèvre, 2012). Muñoz-Jaramillo et al. (2015) proposed that only one component (large sunspots) is generated by the dynamo, while the other component (small sunspots) represents the contribution of the decay process after the magnetic fields emerged. Kostyuchenko (2017) found the difference in the dynamic characteristics of two populations and suggested that while both components could have been generated by the same deep-seated dynamo, a fraction of a large sunspot component could be involved in a secondary process taking place just below the photosphere. Nikbakhsh et al. (2019) studied the solar cycle variability of magnetically simple (SARs) and complex active regions (CARs) and arrived to a similar conclusion that all active regions are formed at the same depth in the solar convection 


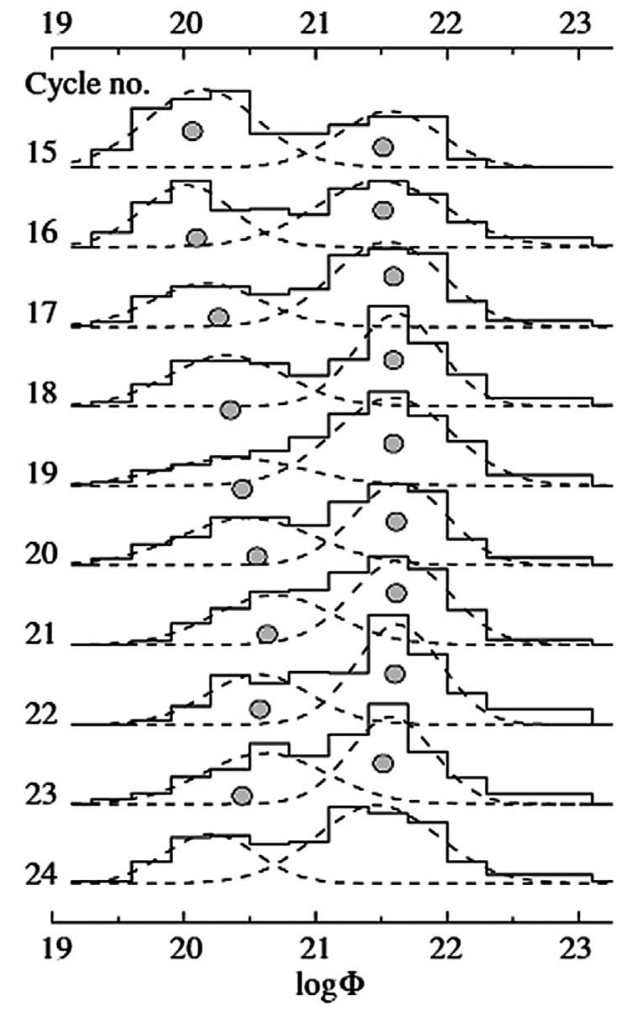

Fig. 14. The PDF of proxy of the magnetic flux $\Phi$ (in units of Maxwell) based on sunspot area for solar cycles 15-24. Dashed lines show the Gaussian fit to two components of sunspot populations, and the filled circles mark the maximum of each fitted distribution. Reproduced from Nagovitsyn et al. (2016).

zone, but some of these regions are later transformed to CARs in the shallow, near the photospheric depths. Tlatov et al. (2019, see, their Fig. 9), however, argued that the bimodal distribution of sunspots represents the different stages of development of the sunspots, with pores and transitional sunspots forming one component of PDF distribution shown in Figure 13, and regular (well-developed) sunspots forming the other.

Whatever is the origin of these two populations of sunspots, the mean properties of their distributions show different long-term variations. Figure 14 shows the PDFs of the magnetic flux of sunspots (Eq. (5)) for solar cycles 15-24. The bimodal distribution of sunspots is clearly present in all cycles. The component associated with the large, long-lived sunspots does not appear to exhibit any significant changes in the distribution of their fluxes. The distribution of magnetic fluxes in small, short-lived sunspots show long-term trends. In cycle 15-16, and later cycle 23-24, we see a larger fraction of small short lived sunspots, and the mean flux in these sunspots is lower than cycles 18-19. The trend is consistent with the centennial (90-100 years) variations characteristic of the Gleissberg cycle.

\section{Magnetic and current helicity}

The recent availability of vector magnetic field magnetograms promoted studies of (magnetic and current) helicity on the Sun and its long-term variations. Magnetic helicity is an integral measure of the topological properties of a magnetic field in a closed volume $V$ :

$$
H_{M}=\int A \cdot B \mathrm{~d} V,
$$

where $A$ is the magnetic vector potential, $B=\nabla \times A$, and $B$ is confined to the volume $V$. Locally, it can be characterized by a number of parameters such as linkage, twist, and writhe of the field lines. In astrophysical dynamos, magnetic helicity is employed as a nonlinear constraint of turbulent generation of large-scale magnetic field (for review, see Brown et al., 1999; Buechner \& Pevtsov, 2003; Pevtsov et al., 2014a; Blackman, 2015).

Computation of magnetic helicity requires knowledge of the vector magnetic field in a volume, but the observations are usually taken in a single layer in the solar atmosphere (i.e., the photosphere). Thus, early studies employed so-called helicity proxies, such as vertical $(z)$ components of current helicity density $J_{z} \cdot B_{z}$ or $\alpha=J_{z} / B_{z}$ (a measure of magnetic twist), and $J$ is the electric current density and $B$ is the magnetic induction. The early studies using active region magnetic fields and chromospheric filaments (e.g., Seehafer, 1990; Martin et al., 1992; Pevtsov et al., 1995; Abramenko et al., 1996, 1997; Bao \& Zhang, 1998; Martin, 1998) led to the establishment of the hemispheric helicity rule, with predominantly negative/positive helicity in the northern/southern hemisphere. Later studies demonstrated that the sign of helicity of large-scale magnetic fields is opposite to the sign of helicity of active regions (Pipin \& Pevtsov, 2014; Brandenburg et al., 2017). The large-scale helicity was also found to evolve during a solar cycle similarly to helicity of active regions.

The solar dynamo theory predicts bi-helical properties of a magnetic field (Seehafer, 1996; Blackman \& Brandenburg, 2002; Brandenburg \& Subramanian, 2005), with the sign of magnetic helicity density of large-scale field corresponding to the sign of the $\alpha$-effect, and the sign of magnetic helicity density of a small-scale field being opposite to the larger-scale field. This is due to the magnetic helicity conservation in a closed volume, when "allocating" the magnetic helicity of one sign to a selected spatial scale should automatically lead to the accumulation of helicity of an opposite sign on the other spatial scale. In the framework of the mean field dynamo models, the small-scale magnetic field corresponds to active regions and the large-scale stands for the global axisymmetric components of solar magnetic activity. Another prediction of mean-field dynamo models includes the existence of polar and equatorial branches in the time-latitude diagram of magnetic helicity evolution, which represent the transport of magnetic helicity flux to the polar regions, both on large and small scales. Following the standard framework of the mean-field magnetohydrodynamics we decompose the magnetic field and its vector-potential into the mean and fluctuating parts: $\mathbf{B}=\overline{\mathbf{B}}+\mathbf{b}, \mathbf{A}=\overline{\mathbf{A}}+\mathbf{a}$, where the small letters represent the small-scale fluctuations and the capital ones with over-bars - the large-scale fields. Similarly to Pipin et al. (2019) we express the relationship between the magnetic helicity density of the large- and small-scale magnetic fields as follows:

$$
\overline{\mathbf{a} \cdot \mathbf{b}}=\overline{\mathbf{A} \cdot \mathbf{B}}-\overline{\mathbf{A}} \cdot \overline{\mathbf{B}} .
$$



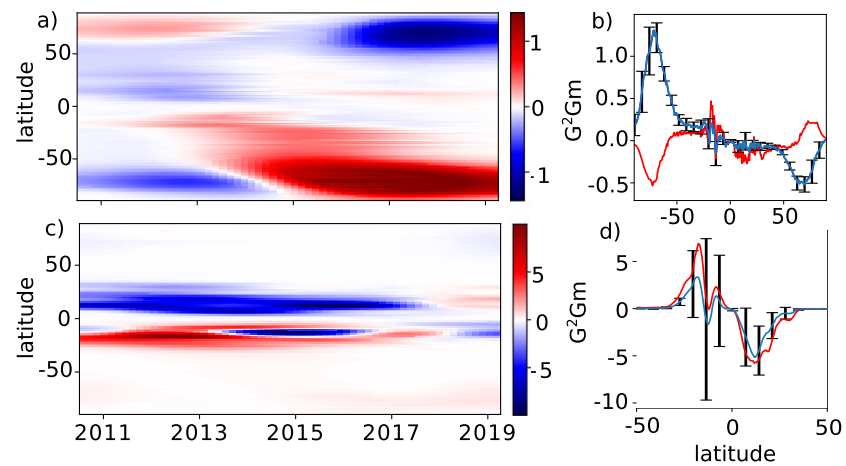

Fig. 15. Magnetic helicity in Solar Cycle 24: (a) The time-latitude evolution of the large-scale magnetic helicity density, $\bar{A} \cdot \bar{B}$; (b) the mean latitudinal profile of the large-scale magnetic helicity density (blue line) with $95 \%$ confidence interval for the standard error. Red line shows the average profile for the first half of the cycle including CR2097-2156; (c) shows the same as (a) for the azimuthal averaging of the small-scale magnetic helicity density $\overline{a \cdot b}$, see equation (11) in Pipin et al. (2019); (d) shows the same as (b) for the $\overline{a \cdot b}$. Used by permission from Pipin et al. (2019).

By definition, the small-scale magnetic helicity density $\overline{\mathbf{a} \cdot \mathbf{b}}$, includes magnetic fields from all range of scales except the axisymmetric magnetic field. Figure 15 provides observational evidence for the hemispheric helicity rule both for large- and small-scales magnetic fields. The presence of polar branches is also seen (Fig. 15a). Small scale helicity has much larger amplitude when compared to large-scale helicity. It's also concentrated in a narrow low-mid- latitude range. This implies that active regions provide the main contribution of helicity on the solar surface. Figure 15 (panels a and b) indicate possible sign-reversal of large-scale magnetic helicity in 2014. The cause of this helicity reversal does not have any theoretical explanation yet. The small scale helicity (Fig. 15, panels c and d) does not exhibit a systematic sign reversal during the same time periods. This study did not clearly show the theoretically predicted opposite sign of magnetic helicity density on neither large nor small scales. Bi-helical properties were studied recently by Brandenburg et al. (2017) and Singh et al. (2018) using the vector magnetic field measurements from HMI/SDO and VSM/SOLIS. The results from these two instruments appear to be inconclusive in respect to the bi-helical nature of solar magnetic fields.

The patterns shown in Figure 15 are partly in agreement with the predictions of the mean-field dynamo, which suggests that the surface distribution of helicity density can be driven by the dynamo processes inside the convection zone. However, the recent analysis of Hawkes \& Yeates (2019) and Yeates (2020) showed that the similar hemispheric helicity rule can result from the linking of magnetic field lines within emerging bipolar regions with an ambient large-scale magnetic field. For the case of simple bipolar regions, this link resolves into the local quadrupole helicity density distribution pattern (see, Figs. 3c and 4c in Hawkes \& Yeates, 2019). A similar pattern was found in the benchmark dynamo model (Pipin et al., 2019, Fig. 1c). Assuming a strict flux balance between the leading and following spots, the emerging bipolar regions does not contribute to the helicity integral. The net helicity flux from this effect is likely to be zero and thus, would have no effect on the hypothesized dynamo saturation inside the convection zone. The impact of the linking of the emerging active regions with global magnetic field on helicity, like the butterfly diagrams shown in Figure 15 require additional studies.

\section{Summary}

In this review paper, we presented a summary of historical, long-term measurements of the magnetic field on the Sun over the last century. The first magnetic field measurements in astrophysics were made in sunspots in 1908. In 1917, long-term measurements of sunspot field strengths begun at MWO, and such systematic measurements still continue at MWO (USA) and CrAO (Russia). Full disk magnetograph measurements, which started in early 1950s, currently continue via observations at (WSO), Global Oscillations Network Group (GONG) instruments, and HMI/SDO. Since October 2017 (SOLIS relocation to Big Bear Solar Observatory), HMI is the only instrument providing full disk vector magnetic fields. Past observations from these and other instruments led to the discovery of several major properties of solar magnetic fields including their polarity and tilt orientation (Hale polarity rule and Joy's law). Still, new analysis of historical data continue to discover new properties of these well-known tendencies. The systematic observations of vector magnetic fields over the entire solar disk enable the exploration of topological properties of magnetic fields that were not previously explored.

We showed that inevitably long-term datasets have nonuniformities related to changes in observation techniques, their observers, and the evolution of our scientific knowledge. In spite of that such historical datasets have an enormous value as they provide information about changes in the processes, which may not have been known at the time the data were taken. Thus, it is absolutely critical that the community recognizes the importance of such long-term datasets, their digitization, and preservation, and provides strong support for the continuation of long-term observations. Otherwise, we may be left without data critical for our understanding and predicting of future solar activity.

\section{Supplementary material}

Supplementary material is available at https://www.swscjournal.org/10.1051/swsc/2020069/olm

Supplementary Movie 1. Southern pole field reversal.

Acknowledgements. The authors acknowledge the financial support by the Academy of Finland to the ReSoLVE Centre of Excellence (project no. 307411), the partial support by NASA (grants 80NSSC17K0686 and NNX15AN43G), Russian Foundation for Basic Research (grants 19-02-00088 and 18-02-00098), support of scientific project FR II.16 of ISTP SB RAS, and the project No 13.1902.21.0039 of the Ministry of Science and Higher Education of the Russian Federation. We acknowledge help by Alexander Pevtsov in preparation of the manuscript. This work utilizes SOLIS and GONG data obtained by the NSO Integrated Synoptic 
Program (NISP), managed by the National Solar Observatory. HMI data used here are courtesy of NASA/SDO and the HMI science teams. Observations from Pulkovo Observatory and early observations from $\mathrm{CrAO}$ are available from the "Combined Database of Sunspot Magnetic Fields" at http://www. gao.spb.ru/database/mfbase/gindex.html. Observations from CrAO for later periods (1983-2019) are available at https://sun.crao.ru/observations/sunspots-magnetic-field. The authors are grateful to the countless observers at Mount Wilson, Crimean, Pulkovo and Potsdam/AIP Observatories, whose dedicated work resulted in the creation of a comprehensive historical dataset of sunspot field strength measurements spanning over more than 100 years. The editor thanks two anonymous reviewers for their assistance in evaluating this paper.

\section{References}

Abramenko VI, Wang T, Yurchishin VB. 1996. Analysis of electric current helicity in active regions on the basis of vector magnetograms. Sol Phys 168(1): 75-89. https://doi.org/10.1007/ BF00145826.

Abramenko VI, Wang T, Yurchishin VB. 1997. Electric current helicity in 40 active regions in the maximum of solar cycle 22. Sol Phys 174: 291-296. https://doi.org/10.1023/A:1004957515498.

Ai G-X, Hu Y-F. 1986. Principles of a solar magnetic field telescope. Acta Astron. Sin. 27: 173-180.

Babcock HW. 1953. The solar magnetograph. Astrophys J 118: 387. https://doi.org/10.1086/145767.

Babcock HD. 1959. The Sun's polar magnetic field. Astrophys J 130: 364. https://doi.org/10.1086/146726.

Babcock HW. 1961. The topology of the Sun's magnetic field and the 22-year cycle. Astrophys J 133: 572. https://doi.org/10.1086/ 147060.

Babcock HW, Babcock HD. 1955. The Sun's magnetic field, 19521954. Astrophys J 121: 349. https://doi.org/10.1086/145994.

Bai XY, Deng YY, Teng F, Su JT, Mao XJ, Wang GP. 2014. Improved magnetogram calibration of solar magnetic field telescope and its comparison with the helioseismic and magnetic imager. Mon Not $R$ Astron Soc 445(1): 49-55. https://doi.org/10.1093/mnras/ stu1711.

Balasubramaniam KS, Pevtsov A. 2011. Ground-based synoptic instrumentation for solar observations. In: Vol. 8148 of Society of Photo-Optical Instrumentation Engineers (SPIE) Conference Series, 814809. https://doi.org/10.1117/12.892824

Bao S, Zhang H. 1998. Patterns of current helicity for the twentysecond solar cycle. Astrophys J Lett 496(1): L43-L46. https://doi. org/10.1086/311232.

Baumann I, Schmitt D, Schüssler M, Solanki SK. 2004. Evolution of the large-scale magnetic field on the solar surface: A parameter study. A\&A 426: 1075-1091. https://doi.org/10.1051/0004-6361: 20048024.

Berger TE, Lites BW. 2002. Weak-field magnetogram calibration using advanced Stokes polarimeter flux-density maps - I. Solar optical universal polarimeter calibration. Sol Phys 208(2): 181210. https://doi.org/10.1023/A:1020537923728.

Berger TE, Lites BW. 2003. Weak-field magnetogram calibration using advanced Stokes polarimeter flux density maps - II. SOHO/ MDI full-disk mode calibration. Sol Phys 213(2): 213-229. https://doi.org/10.1023/A:1023953716633.
Bertello L, Pevtsov AA, Petrie GJD, Keys D. 2014. Uncertainties in solar synoptic magnetic flux maps. Sol Phys 289(7): 2419-2431. https://doi.org/10.1007/s11207-014-0480-3.

Blackman EG. 2015. Magnetic helicity and large scale magnetic fields: A primer. Space Sci Rev 188: 59-91. https://doi.org/ 10.1007/s11214-014-0038-6.

Blackman EG, Brandenburg A. 2002. Dynamic nonlinearity in largescale dynamos with shear. Astrophys J 579(1): 359-373. https://doi.org/10.1086/342705.

Blanco Rodríguez J, del Toro Iniesta JC, Orozco Suárez D, Martnez Pillet V, Bonet JA, Feller A, Hirzberger J, Lagg A, Piqueras J, Gasent Blesa JL. 2018. SOPHISM: An end-to-end software instrument simulator. Astrophys J Suppl Ser 237(2): 35. https://doi. org/10.3847/1538-4365/aad242.

Borrero JM, Tomczyk S, Kubo M, Socas-Navarro H, Schou J, Couvidat S, Bogart R. 2011. VFISV: Very fast inversion of the Stokes vector for the helioseismic and magnetic imager. Sol Phys 273(1): 267-293. https://doi.org/10.1007/s11207-0109515-6.

Brandenburg A, Subramanian K. 2005. Astrophysical magnetic fields and nonlinear dynamo theory. Phys Rep 417(1-4): 1-209. https://doi.org/10.1016/j.physrep.2005.06.005.

Brandenburg A, Petrie GJD, Singh NK. 2017. Two-scale analysis of solar magnetic helicity. Astrophys J 836(1): 21. https://doi.org/ 10.3847/1538-4357/836/1/21.

Brouwer MP, Zwaan C. 1990. Sunspot nests as traced by a cluster analysis. Sol Phys 129(2): 221-246. https://doi.org/10.1007/ BF00159038.

Brown MT, Canfield RC, Pevtsov AA. (eds). 1999. Magnetic helicity in space abnd laboratory plasmas, no. 111 in Geophysical Monograph, AGU, Washington, DC.

Buechner J, Pevtsov AA (Eds.). 2003. Magnetic Helicity at the Sun, in Solar Wind and Magnetospheres; Vistas from X-Ray Observatories. Advances in Space Research 32(10): 1817-1999. https://doi.org/10.1016/S0273-1177(03)90615-6.

Bumba V, Howard R. 1969. Solar activity and recurrences in magnetic-field distribution. Sol Phys 7(1): 28-38. https://doi.org/ 10.1007/BF00148402.

Cacciani A, Varsik J, Zirin H. 1990. Observations of vector magnetic fields with a magneto-optic filter. Sol Phys 125: 173-178. https://doi.org/10.1007/BF00154786.

Carrington CR. 1863. Observations of the spots on the Sun from November 9, 1853, to March 24, 1861, made at Redhill, Williams and Norgate, London.

Centeno R, Schou J, Hayashi K, Norton A, Hoeksema JT, Liu Y, Leka KD, Barnes G. 2014. The helioseismic and magnetic imager (HMI) vector magnetic field pipeline: Optimization of the spectral line inversion code. Sol Phys 289(9): 3531-3547. https://doi.org/ 10.1007/s11207-014-0497-7.

Cho IH, Cho KS, Bong SC, Lim EK, Kim RS, Choi S, Kim YH, Yurchyshyn V. 2015. Statistical comparison between pores and sunspots by using SDO/HMI. Astrophys J 811(1): 49. https://doi. org/10.1088/0004-637X/811/1/49.

Clette F, Lefèvre L. 2012. Are the sunspots really vanishing? Anomalies in solar cycle 23 and implications for long-term models and proxies. J Space Weather Space Clim 2: A06. https://doi.org/ 10.1051/swsc/2012007.

Clette F, Svalgaard L, Vaquero JM, Cliver EW. 2014. Revisiting the sunspot number. A 400-year perspective on the solar cycle. Space Sci Rev 186(1-4): 35-103. https://doi.org/10.1007/s11214-014-0074-2.

Cliver EW. 2014. The extended cycle of solar activity and the Sun's 22-year magnetic cycle. Space Sci Rev 186(1-4): 169-189. https://doi.org/10.1007/s11214-014-0093-z. 
Cortie AL. 1892. Observations of the spectra of the sun-spots in the region B-D, made at the Stonyhurst college observatory. MmRAS 50: 29.

Cortie AL. 1898. Vanadium in the spectrum (C to D) of sun-spots. Mon Not R Astron Soc 58: 370. https://doi.org/10.1093/mnras/58.7.370.

Cortie AL. 1904. The spectra of Sun-spots in the red and yellow regions of the spectrum. Astrophys J 20: 253. https://doi.org/ $10.1086 / 141160$.

Couvidat S, Schou J, Hoeksema JT, Bogart RS, Bush RI, Duvall TL, Liu Y, Norton AA, Scherrer PH. 2016. Observables processing for the helioseismic and magnetic imager instrument on the solar dynamics observatory. Sol Phys 291(7): 1887-1938. https://doi. org/10.1007/s11207-016-0957-3.

Fan Y, Fisher GH, Deluca EE. 1993. The origin of morphological asymmetries in bipolar active regions. Astrophys $J$ 405: 390. https://doi.org/10.1086/172370.

Fisher GH, Fan Y, Howard RF. 1995. Comparisons between theory and observation of active region tilts. Astrophys $J$ 438: 463. https://doi.org/10.1086/175090.

Fisher GH, Fan Y, Longcope DW, Linton MG, Pevtsov AA. 2000. The solar dynamo and emerging flux - (invited review). Sol Phys 192: 119-139. https://doi.org/10.1023/A:1005286516009.

Gosain S, Pevtsov AA, Rudenko GV, Anfinogentov SA. 2013. First synoptic maps of photospheric vector magnetic field from SOLIS/ VSM: Non-radial magnetic fields and hemispheric pattern of helicity. Astrophys J 772(1): 52. https://doi.org/10.1088/0004637X/772/1/52.

Grigoryev VM, Kobanov NI, Osak BF, Selivanov VL, Stepanov VE. 1985. The vector magnetograph of the Sayan Solar Observatory. In: Measurements of solar vector magnetic fields, Hagyard MJ (Ed.), NASA Conference Proceedings 2375, NASA, Washington, DC, pp. 231-256.

Grotrian W. 1953. Polaritäten und Maximalwerte magnetischer Feldstärken von Sonnenflecken in den Jahren 1946-1951. Publikationen des Astrophysikalischen Observatoriums zu Potsdam 29 (97): DI-D160.

Grotrian W. 1956. Polaritäten und Maximalwerte magnetischer Feldstärken von Sonnenflecken in den Jahren 1952-1953. Publikationen des Astrophysikalischen Observatoriums zu Potsdam 30(99): $1-42$.

Gyenge N, Ludmány A, Baranyi T. 2016. Active longitude and solar flare occurrences. Astrophys J 818(2): 127. https://doi.org/ 10.3847/0004-637X/818/2/127.

Hagino M, Nakatani Y, Ishii TT, Hanaoka Y, Sakurai T, Hiei E, Suzuki D. 2009. Comparison of the Vector Magnetograms taken with the SFT/MTK and the SST/KSW. In: Solar Polarization 5, Berdyugina SV, Nagendra KN, Ramelli R (Eds.), Vol. 405 of Astronomical Society of the Pacific Conference Series, 393.

Hagyard MJ (Ed.). 1985. Measurements of solar vector magnetic fields. In: NASA Conference Proceedings 2375, Hagyard MJ (Eds.), NASA, Washington, DC, pp. 1-470. ADS: https://ui. adsabs.harvard.edu/abs/1985svmf.nasa.....H/abstract.

Hagyard MJ, Cumings NP, West EA, Smith JE. 1982. The MSFC vector magnetograph. Sol Phys 80: 33-51. https://doi.org/10.1007/ BF00153422.

Hale GE. 1908. Solar vortices and the Zeeman effect. PASP 20(121): 220. https://doi.org/10.1086/121822.

Hale GE. 1912. Preliminary note on an attempt to detect the general magnetic field of the Sun. Terr Magn Atmos Electr (J Geophys Res) 17(4): 173. https://doi.org/10.1029/TE017i004p00173.

Hale GE. 1913. Preliminary results of an attempt to detect the general magnetic field of the Sun. Astrophys J 38: 27. https://doi.org/ $10.1086 / 142013$.
Hale GE, Nicholson SB. 1925. The law of Sun-spot polarity. Astrophys J 62: 270. https://doi.org/10.1086/142933.

Hale GE, Seares FH, van Maanen A, Ellerman F. 1918. The general magnetic field of the Sun. Apparent variation of field-strength with level in the solar atmosphere. Astrophys $J$ 47: 206. https://doi.org/ $10.1086 / 142403$.

Hale GE, Ellerman F, Nicholson SB, Joy AH. 1919. The magnetic polarity of Sun-spots. Astrophys J 49: 153. https://doi.org/10.1086/142452.

Harker BJ. 2017. VFISV inversion code documentation for SOLIS/VSM pipeline implementation, National Solar Observatory Technical Report No. NSO/NISP-2017-02, arXiv e-prints, arXiv:1707.00002.

Hathaway DH. 2015. The Solar cycle. Living Rev Sol Phys 12(1): 4. https://doi.org/10.1007//rsp-2015-4.

Hathaway DH, Upton L. 2014. The solar meridional circulation and sunspot cycle variability. J Geophys Res (Space Phys) 119(5): 3316-3324. https://doi.org/10.1002/2013JA019432.

Hawkes G, Yeates AR. 2019. Hemispheric injection of magnetic helicity by surface flux transport. $A \& A$ 631: A138. https://doi.org/ 10.1051/0004-6361/201936475.

Hoeksema JT, Liu Y, Hayashi K, Sun X, Schou J, et al. 2014. The helioseismic and magnetic imager (HMI) vector magnetic field pipeline: Overview and performance. Sol Phys 289(9): 3483-3530. https://doi.org/10.1007/s11207-014-0516-8.

Hofmann A, Grigor'ev VM, Selivanov VL. 1988a. Analysis and results of cooperative magnetographic measurements. II - Comparison and interpretation of the current densities. Astron Nachr 309(6): 373-379. https://doi.org/10.1002/asna.2113090608.

Hofmann A, Grigor'ev VM, Selivanov VL, Klvana M. 1988b. Analysis and results of cooperative magnetographic measurements. I - Correction, comparison and discussion of measurements. Astron Nachr 309(5): 331-340. https://doi.org/10.1002/asna.2113090510.

Holder ZA, Canfield RC, McMullen RA, Nandy D, Howard RF, Pevtsov AA. 2004. On the tilt and twist of solar active regions. Astrophys J 611(2): 1149-1155. https://doi.org/10.1086/422247.

Houtgast J, van Sluiters A. 1948. Statistical investigations concerning the magnetic fields of sunspots I. Bull Astron Inst Netherlands 10: 325.

Howard R. 1963. On the relation of major solar flares with changes in sunspot areas. Astrophys J 138: 1312. https://doi.org/10.1086/147730.

Howard R. 1974. Studies of solar magnetic fields. I: The average field strengths. Sol Phys 38(2): 283-299. https://doi.org/10.1007/ BF00155067.

Howard R. 1985. Eight decades of solar research at Mount Wilson. Sol Phys 100: 171-187. https://doi.org/10.1007/BF00158427.

Howard R, Bumba V, Smith SF. 1967. Atlas of solar magnetic fields, Carnegie Institution of Washington Publication No. 626, Carnegie Institution of Washington, Washington, DC. https://ui.adsabs. harvard.edu/abs/1967asmf.book.....H/abstract.

Ichimoto K, Sakurai T, Hiei E, Nishino Y, Shinoda K, et al. 1993. Solar flare telescope project. Rep Nat Astron Obs Jpn 1(4): 375-390.

Ikhsanov RN. 1968. The observation and interpretation of some regularities of sunspot development. Soviet Ast 11: 843.

Illarionov E, Tlatov A, Sokoloff D. 2015. The properties of the tilts of bipolar solar regions. Sol Phys 290(2): 351-361. https://doi.org/ 10.1007/s11207-014-0612-9.

Jones H. 1993. Spectrometer-based magnetographs (invited). In: IAU Colloq. 141: The magnetic and velocity fields of solar active regions, Zirin H, Ai G, Wang H (Eds.), Vol. 46 of Astronomical Society of the Pacific Conference Series, 156.

Jones HP, Ceja JA. 2001. Preliminary comparison of magnetograms from KPVT/SPM, SOHO/MDI and $\mathrm{GONG}^{+}$. In: Advanced solar polarimetry - Theory, observation, and instrumentation, Sigwarth M (Ed.), Vol. 236 of Astronomical Society of the Pacific Conference Series, 87. 
Jones HP, Duvall J, Thomas L, Harvey JW, Mahaffey CT, Schwitters JD, Simmons JE. 1992. The NASA/NSO spectromagnetograph. Sol Phys 139(2): 211-232. https://doi.org/10.1007/BF00159149.

Keller CU, Harvey JW, Giampapa MS. 2003. SOLIS: An innovative suite of synoptic instruments. In: Innovative telescopes and instrumentation for solar astrophysics, Keil SL, Avakyan SV (Eds.), Vol. 4853 of Proc. SPIE, 194-204. https://doi.org/10.1117/12.460373.

Kleeorin N, Safiullin N, Kuzanyan K, Rogachevskii I, Tlatov A, Porshnev S. 2020. The mean tilt of sunspot bipolar regions: Theory, simulations and comparison with observations. Mon Not $R$ Astron Soc 495: 238-248. https://doi.org/10.1093/mnras/staa1047.

Kosovichev AG, Stenflo JO. 2008. Tilt of emerging bipolar magnetic regions on the Sun. Astrophys J Lett 688(2): L115. https://doi.org/ $10.1086 / 595619$.

Kostyuchenko IG. 2017. Dynamic characteristics of area variations of small and large sunspots and quasi-Biennial oscillations in solar activity. Geomagn Aeron 57(7): 814-820. https://doi.org/10.1134/ S0016793217070118.

Kotov VA, Mozzerin VM. 1988. Obituary: Andrei Borisovich Severny. Sol Phys 115(1): 1-3. https://doi.org/10.1007/BF00146225.

Kuklin GV. 1973. On two populations of sunspot groups. Byulletin Solnechnye Dannye Akademie Nauk SSSR 1973: 53-61.

Kuklin GV. 1980. On two populations of sunspot groups. Bull Astron Inst Czech 31: 224.

Künzel H, Mattig W, Schröter EH. 1956a. Polaritäten und Maximalwerte magnetischer Feldstärken von Sonnenflecken im Zeitraum 1956 April I bis Juni 30. Astron Nachr 283(4): 159. https://doi.org/10.1002/asna.19562830406.

Künzel H, Mattig W, Schröter EH. 1956b. Polaritäten und Maximalwerte magnetischer Feldstärken von Sonnenflecken in dem Zeitraum 1956 Januar I-März 31. Astron Nachr 283(2): 89. https://doi.org/10.1002/asna.19562830209.

Künzel H, Mattig W, Schröter EH. 1956c. Polaritäten und Maximalwerte magnetischer Feldstärken von Sonnenflecken in den Jahren 1954-1955. Astron Nachr 283(1): 28. https://doi.org/ 10.1002/asna.19562830107.

Künzel H, Mattig W, Schröter EH. 1957. Polaritäten und Maximalwerte magnetischer Feldstärken von Sonnenflecken im Zeitraum 1956 Juli I bis August 31. Astron Nachr 283(6): 251. https://doi. org/10.1002/asna.19562830605.

Kuzanyan KM, Safiullin N, Kleeorin N, Rogachevskii I, Porshnev S. 2019. Large-scale properties of the tilt of sunspot groups and Joy's law near the solar equator. Astrophysics 62(2): 261-275. https://doi.org/10.1007/s10511-019-09579-2.

Lefèvre L, Clette F. 2011. A global small sunspot deficit at the base of the index anomalies of solar cycle 23. A\&A 536: L11. https://doi.org/10.1051/0004-6361/201118034.

Leighton RB. 1969. A magneto-kinematic model of the solar cycle. Astrophys J 156: 1. https://doi.org/10.1086/149943.

Leka KD, Barnes G, Crouch AD, Metcalf TR, Gary GA, Jing J, Liu Y. 2009. Resolving the 180 ambiguity in solar vector magnetic field data: Evaluating the effects of noise, spatial resolution, and method assumptions. Sol Phys 260(1): 83-108. https://doi.org/ 10.1007/s11207-009-9440-8.

Li J. 2018. A systematic study of hale and anti-hale sunspot physical parameters. Astrophys J 867(2): 89. https://doi.org/10.3847/15384357/aae31a.

Li J, Ulrich RK. 2012. Long-term measurements of sunspot magnetic tilt angles. Astrophys J 758(2): 115. https://doi.org/10.1088/0004637X/758/2/115.

Lites BW, Akin DL, Card G, Cruz T, Duncan DW, et al. 2013. The Hinode Spectro-Polarimeter. Sol Phys 283(2): 579-599. https://doi. org/10.1007/s11207-012-0206-3.
Liu Y, Hoeksema JT, Scherrer PH, Schou J, Couvidat S, Bush RI, Duvall TL, Hayashi K, Sun X, Zhao X. 2012. Comparison of lineof-sight magnetograms taken by the solar dynamics observatory/ helioseismic and magnetic imager and solar and heliospheric observatory/Michelson Doppler imager. Sol Phys 279(1): 295316. https://doi.org/10.1007/s11207-012-9976-x.

Livingston W, Harvey J. 1971. The Kitt Peak Magnetograph. Iv: 40CHANNEL probe and the detection of weak photospheric fields. In: Solar magnetic fields, Howard R (Ed.), Vol. 43 of IAU Symposium, $51 \mathrm{p}$.

Livingston W, Watson F. 2015. A new solar signal: Average maximum sunspot magnetic fields independent of activity cycle. Geophys Res Lett 42(21): 9185-9189. https://doi.org/10.1002/2015GL065413.

Livingston W, Harvey J, Slaughter C. 1971. The Kitt Peak Magnetograph, Iii: Automation and the 40-CHANNEL probe. Publ R Obs Edinburgh 8: 52.

Livingston WC, Harvey J, Slaughter C, Trumbo D. 1976. Solar magnetograph employing integrated diode arrays. Appl Opt 15: 40-52. https://doi.org/10.1364/AO.15.000040.

Livingston W, Harvey JW, Malanushenko OV, Webster L. 2006. Sunspots with the strongest magnetic fields. Sol Phys 239(1-2): 41-68. https://doi.org/10.1007/s11207-006-0265-4.

Livingston W, Penn MJ, Svalgaard L. 2012. Decreasing sunspot magnetic fields explain unique $10.7 \mathrm{~cm}$ radio flux. Astrophys $J$ Lett 757(1): L8. https://doi.org/10.1088/2041-8205/757/1/L8.

Lockyer JN. 1867. Spectroscopic observations of the sun. Proc $R$ Soc Lond 15: 256-258. https://doi.org/10.1098/rspl.1866.0059.

Longcope D, Linton M, Pevtsov A, Fisher G, Klapper I. 1999. Twisted flux tubes and how they get that way. Washington DC Am Geophys Union Geophys Monogr Ser 111: 93-101. https://doi.org/ 10.1029/GM111p0093.

López Fuentes MC, Demoulin P, Mandrini CH, van Driel-Gesztelyi L. 2000. The counterkink rotation of a non-hale active region. Astrophys J 544(1): 540-549. https://doi.org/10.1086/317180.

Lozitska NI, Lozitsky VG, Andryeyeva OA, Akhtemov ZS, Malashchuk VM, Perebeynos VA, Stepanyan NN, Shtertser NI. 2015. Methodical problems of magnetic field measurements in umbra of sunspots. Adv Space Res 55(3): 897-907. https://doi.org/ 10.1016/j.asr.2014.08.006.

Lundstedt H, Persson T, Andersson V. 2015. The extreme solar storm of May 1921: Observations and a complex topological model. Ann Geophys 33(1): 109-116. https://doi.org/10.5194/angeo-33-109-2015.

Makita M, Hamana S, Nishi K. 1985a. The solar vector magnetograph of the Okayama Astrophysical Observatory. In: Measurements of solar vector magnetic fields, Hagyard MJ (Ed.), Vol. 2375 of NASA Conference Proceedings, NASA, pp. 173-182.

Makita M, Nishi K, Shimizu M, Hamana S, Sakurai T, Grigoryev VM, Kuklin GV, Selivanov VL. 1985b. Comparisons of simultaneous vector magnetograms. In: Measurements of solar vector magnetic fields, Hagyard MJ (Ed.), pp. 399-402.

Martin SF. 1998. Conditions for the formation and maintenance of filaments (invited review). Sol Phys 182(1): 107-137. https://doi. org/10.1023/A:1005026814076.

Martin SF, Marquette WH, Bilimoria R. 1992. The solar cycle pattern in the direction of the magnetic field along the long axes of polar filaments. In: The solar cycle, Harvey KL (Ed.), Vol. 27 of Astronomical Society of the Pacific Conference Series, 53 p.

Mártinez Pillet V, Collados M, Sánchez Almeida J, González V, Cruz-Lopez A, et al. 1999. LPSP \& TIP: Full Stokes Polarimeters for the Canary Islands Observatories. In: High Resolution Solar Physics: Theory, Observations, and Techniques, Rimmele TR, Balasubramaniam KS, Radick RR (Eds.), Vol. 183 of Astronomical Society of the Pacific Conference Series, 264 p. 
Maunder EW. 1904. Note on the distribution of sun-spots in heliographic latitude, 1874-1902. Mon Not R Astron Soc 64: 747-761. https://doi.org/10.1093/mnras/64.8.747.

McClintock BH, Norton AA. 2016. Tilt angle and footpoint separation of small and large bipolar sunspot regions observed with HMI. Astrophys J 818(1): 7. https://doi.org/10.3847/0004637X/818/1/7.

McClintock BH, Norton AA, Li J. 2014. Re-examining sunspot tilt angle to include anti-hale statistics. Astrophys $J$ 797(2): 130. https://doi.org/10.1088/0004-637X/797/2/130.

Metcalf TR. 1994. Resolving the 180-degree ambiguity in vector magnetic field measurements: The "minimum" energy solution. Sol Phys 155(2): 235-242. https://doi.org/10.1007/BF00680593.

Metcalf TR, Leka KD, Barnes G, Lites BW, Georgoulis MK, et al. 2006. An overview of existing algorithms for resolving the 180 ambiguity in vector magnetic fields: Quantitative tests with synthetic data. Sol Phys 237(2): 267-296. https://doi.org/ 10.1007/s11207-006-0170-x.

Mickey DL. 1985. The Haleakala Stokes polarimeter. Sol Phys 97: 223-238. https://doi.org/10.1007/BF00165987.

Mickey DL, Canfield RC, Labonte BJ, Leka KD, Waterson MF, Weber HM. 1996. The imaging vector magnetograph at Haleakala. Sol Phys 168(2): 229-250. https://doi.org/10.1007/BF00148052.

Mitchell WM. 1904. Reversals in the spectra of sun-spots. Astrophys $J$ 19: 357. https://doi.org/10.1086/141125.

Mitchell WM. 1905. Researches in the sun-spot spectrum, region $\mathrm{F}$ to $\alpha$. Astrophys J 22: 4. https://doi.org/10.1086/141237.

Mitchell WM. 1906. Results of solar observations at Princeton, 1905-1906. Astrophys J 24: 78. https://doi.org/10.1086/141375.

Mordvinov A, Pevtsov A, Bertello L, Petri G. 2016. The reversal of the Sun's magnetic field in cycle 24. Sol-Terr Phys 2(1): 3-18. https://doi.org/10.12737/16356.

Muñoz-Jaramillo A, Senkpeil RR, Windmueller JC, Amouzou EC, Longcope DW, et al. 2015. Small-scale and global dynamos and the area and flux distributions of active regions, sunspot groups, and sunspots: A multi-database study. Astrophys $J$ 800(1): 48 . https://doi.org/10.1088/0004-637X/800/1/48.

Nagovitsyn YA, Pevtsov AA. 2016. On the presence of two populations of sunspots. Astrophys $J$ 833(1): 94. https://doi.org/ 10.3847/1538-4357/833/1/94.

Nagovitsyn YA, Pevtsov AA, Livingston WC. 2012. On a possible explanation of the long-term decrease in sunspot field strength. Astrophys J Lett 758(1): L20. https://doi.org/10.1088/2041-8205/ 758/1/L20.

Nagovitsyn YA, Pevtsov AA, Osipova AA, Tlatov AG, Miletskii EV, Nagovitsyna EY. 2016. Two populations of sunspots and secular variations of their characteristics. Astron Lett 42(10): 703-712. https://doi.org/10.1134/S1063773716090048.

Nagovitsyn YA, Pevtsov AA, Osipova AA. 2017. Long-term variations in sunspot magnetic field-area relation. Astron Nachr 338(1): 26-34. https://doi.org/10.1002/asna.201613035.

Nagovitsyn YA, Pevtsov AA, Osipova AA, Ivanov VG. 2018. Some features of the two sunspot group populations' properties. Geomagn Aeron 58(8): 1170-1174. https://doi.org/10.1134/ S001679321808025X.

Nicholson SB. 1933. The area of a sun-spot and the intensity of its magnetic field. PASP 45(263): 51-53. https://doi.org/10.1086/ 124301.

Nikbakhsh S, Tanskanen EI, Käpylä MJ, Hackman T. 2019. Differences in the solar cycle variability of simple and complex active regions during 1996-2018. A\&A 629: A45. https://doi.org/ 10.1051/0004-6361/201935486.
Norton AA, Gilman PA. 2005. Recovering solar toroidal field dynamics from sunspot location patterns. Astrophys $J$ 630(2): 1194-1205. https://doi.org/10.1086/431961.

Norton AA, Graham JP, Ulrich RK, Schou J, Tomczyk S, et al. 2006. Spectral line selection for HMI: A comparison of Fe I $6173 \AA$ and Ni I 6768 Å. Sol Phys 239(1-2): 69-91. https://doi.org/10.1007/ s11207-006-0279-y.

Okamoto TJ, Sakurai T. 2018. Super-strong magnetic field in sunspots. Astrophys J Lett 852(1): L16. https://doi.org/10.3847/ 2041-8213/aaa3d8.

Penn MJ, Livingston W. 2006. Temporal changes in sunspot umbral magnetic fields and temperatures. Astrophys J Lett 649(1): L45-L48. https://doi.org/10.1086/508345.

Penn MJ, Livingston W. 2011. Long-term evolution of sunspot magnetic fields. In: Physics of Sun and Star Spots, Prasad Choudhary D, Strassmeier KG (Eds.), Vol. 273 of IAU Symposium, pp. 126-133. https://doi.org/10.1017/S1743921311015122.

Petrie G. 2017. High-resolution vector magnetograms of the Sun's poles from Hinode: Flux distributions and global coronal modeling. Sol Phys 292(1): 13. https://doi.org/10.1007/s11207-016-1034-7.

Petrovay K. 2020. Solar cycle prediction. Living Rev Sol Phys 17(1): 2. https://doi.org/10.1007/s41116-020-0022-z.

Petrovay K, Talafha M. 2019. Optimization of surface flux transport models for the solar polar magnetic field. A\&A 632: A87. https://doi.org/10.1051/0004-6361/201936099.

Pevtsov AA, Canfield RC, Metcalf TR. 1995. Latitudinal variation of helicity of photospheric magnetic fields. Astrophys J Lett 440 L109. https://doi.org/10.1086/187773.

Pevtsov AA, Nagovitsyn YA, Tlatov AG, Rybak AL. 2011. Longterm trends in sunspot magnetic fields. Astrophys $J$ Lett 742(2): L36. https://doi.org/10.1088/2041-8205/742/2/L36.

Pevtsov AA, Berger MA, Nindos A, Norton AA, van Driel-Gesztelyi L. 2014a. Magnetic helicity, tilt, and twist. Space Sci Rev 186(1-4): 285-324. https://doi.org/10.1007/s11214-014-0082-2.

Pevtsov AA, Bertello L, Tlatov AG, Kilcik A, Nagovitsyn YA, Cliver EW. 2014b. Cyclic and long-term variation of sunspot magnetic fields. Sol Phys 289(2): 593-602. https://doi.org/ 10.1007/s11207-012-0220-5.

Pevtsov AA, Virtanen I, Mursula K, Tlatov A, Bertello L. 2016. Reconstructing solar magnetic fields from historical observations. I. Renormalized Ca K spectroheliograms and pseudo-magnetograms. A\&A 585: A40. https://doi.org/10.1051/0004-6361/201526620.

Pevtsov AA, Tlatova KA, Pevtsov AA, Heikkinen E, Virtanen I, Karachik NV, Bertello L, Tlatov AG, Ulrich R, Mursula K. 2019a. Database of Sunspot magnetic field and polarity measurements at Mount Wilson Observatory 1917-2016. https://doi.org/10.25668/ bkt9-4d24.

Pevtsov AA, Tlatova KA, Pevtsov AA, Heikkinen E, Virtanen I, Karachik NV, Bertello L, Tlatov AG, Ulrich R, Mursula K. 2019b. Reconstructing solar magnetic fields from historical observations. V. Sunspot magnetic field measurements at Mount Wilson Observatory. A\&A 628: A103. https://doi.org/10.1051/0004-6361/ 201834985.

Pflug K, Grigoryev VM. 1986. The chain of the solar magnetographs and its results. Contr Astron Obs Skalnate Pleso 15: 453-468.

Pierce AK. 1969. The solar program of the Kitt Peak National Observatory. Sol Phys 6(3): 498-503. https://doi.org/10.1007/ BF00146486.

Pietarila A, Bertello L, Harvey JW, Pevtsov AA. 2013. Comparison of ground-based and space-based longitudinal magnetograms. Sol Phys 282(1): 91-106. https://doi.org/10.1007/s11207-0120138-y. 
Pipin VV, Pevtsov AA. 2014. Magnetic helicity of the global field in solar cycles 23 and 24. Astrophys J 789(1): 21. https://doi.org/ 10.1088/0004-637X/789/1/21.

Pipin VV, Pevtsov AA, Liu Y, Kosovichev AG. 2019. Evolution of magnetic helicity in solar cycle 24. Astrophys J Lett 877(2): L36. https://doi.org/10.3847/2041-8213/ab21bf.

Plotnikov A, Kutsenko A. 2018. Deriving absolute value of the magnetic field strength from I and V stokes parameters. Izvestiya Krymskoi Astrofizicheskoi Observatorii 114(2): 87-96. https://doi. org/10.31059/izcrao-vol114-iss2-pp87-96.

Plowman J, Berger T. 2020. Calibrating GONG magnetograms with end-to-end instrument simulation III: Comparison, calibration, and results. arXiv e-prints, arXiv:2002.02486.

Poisson M, López Fuentes MC, Mandrini CH, Démoulin P, MacCormack C. 2020. Correcting the effect of magnetic tongues on the tilt angle of bipolar active regions. A\&A 633: A151. https://doi.org/10.1051/0004-6361/201936924.

Povel HP. 2001. Ground-based instrumentation for solar magnetic field studies, with special emphasis on the Zurich imaging polarimeters ZIMPOL-I and II. In: Magnetic fields across the HertzsprungRussell diagram, Mathys G, Solanki SK, Wickramasinghe DT (Eds.), Vol. 248 of Astronomical Society of the Pacific Conference Series, $543 \mathrm{p}$.

Report of Committee on Sun-Spot Spectra. 1908. In Transactions of the International Union for Cooperation in Solar Research, Vol. 2, University Press, Manchester, 211 p. URL https://books.google. com/books/about/Transactions_of_the_International_Union.html? id=pDO4AAAAIAAJ.

Rezaei R, Beck C, Schmidt W. 2012. Variation in sunspot properties between 1999 and 2011 as observed with the Tenerife Infrared Polarimeter. A\&A 541: A60. https://doi.org/10.1051/0004-6361/ 201118635.

Riley P, Ben-Nun M, Linker JA, Mikic Z, Svalgaard L, Harvey J, Bertello L, Hoeksema T, Liu Y, Ulrich R. 2014. A multi-observatory inter-comparison of line-of-sight synoptic solar magnetograms. Sol Phys 289(3): 769-792. https://doi.org/10.1007/s11207-013-0353-1.

Ringnes TS. 1965. Secular variations in the magnetic field strength of sunspot groups. Astrophys Norv 10: 27.

Ringnes TS, Jensen E. 1960. On the relation between magnetic fields and areas of sunspots in the interval 1917-56. Astrophys Norv 7: 99.

Ronan RS, Orrall FQ, Mickey DL, West EA, Hagyard MJ, Balasubramaniam KS. 1992. A comparison of vector magnetograms from the marshall space flight center and mees solar observatory. Sol Phys 138(1): 49-68. https://doi.org/10.1007/BF00146196.

Rudenko GV, Anfinogentov SA. 2014. Very fast and accurate Azimuth disambiguation of vector magnetograms. Sol Phys 289(5): 1499-1516. https://doi.org/10.1007/s11207-013-0437-y.

Sainz Dalda A. 2017. A statistical comparison between photospheric vector magnetograms obtained by SDO/HMI and Hinode/SP. Astrophys J 851(2): 111. https://doi.org/10.3847/1538-4357/aa97e3.

Sankarasubramanian K, Lites B, Gullixson C, Elmore D, Hegwer S, Streander K, Rimmele T, Fletcher S, Gregory S, Sigwarth M. 2006. The diffraction limited spectro-polarimeter. In: Solar polarization 4, Casini R, Lites BW (Eds.), Vol. 358 of Astronomical Society of the Pacific Conference Series, pp. 201.

Schatten KH, Scherrer PH, Svalgaard L, Wilcox JM. 1978. Using dynamo theory to predict the sunspot number during solar cycle 21. Geophys Res Lett 5: 411-414. https://doi.org/10.1029/ GL005i005p00411.

Scherrer PH, Wilcox JM, Svalgaard L, Duvall TL, Dittmer PH, Gustafson EK. 1977. The mean magnetic field of the Sun: Observations at Stanford. Sol Phys 54(2): 353-361. https://doi.org/ 10.1007/BF00159925.
Scherrer PH, Bogart RS, Bush RI, Hoeksema JT, Kosovichev AG, et al. 1995. The solar oscillations investigation - Michelson Doppler imager. Sol Phys 162(1-2): 129-188. https://doi.org/ 10.1007/BF00733429.

Scherrer PH, Schou J, Bush RI, Kosovichev AG, Bogart RS, et al. 2012. The helioseismic and magnetic imager (HMI) Investigation for the solar dynamics observatory (SDO). Sol Phys 275: 207-227. https://doi.org/10.1007/s11207-011-9834-2.

Schröter EH. 1953. Ein Versuch zur Bestimmung des Verlaufes der magnetischen Feldstärke über die Fläche eines Sonnenflekes. Mit 5 Textabbildungen. ZAp 33: 20.

Seehafer N. 1990. Electric current helicity in the solar atmosphere. Sol Phys 125(2): 219-232. https://doi.org/10.1007/BF00158402.

Seehafer N. 1996. Nature of the $\alpha$ effect in magnetohydrodynamics. Phys Rev E 53(1): 1283-1286. https://doi.org/10.1103/ PhysRevE.53.1283.

Severny AB. 1965. Introductory report. In: Stellar and solar magnetic fields, Lust R (Ed.), Vol. 22 of IAU Symposium, pp. 238.

Singh NK, Käpylä MJ, Brandenburg A, Käpylä PJ, Lagg A, Virtanen I. 2018. Bihelical spectrum of solar magnetic helicity and its evolution. Astrophys J 863(2): 182. https://doi.org/10.3847/1538-4357/aad0f2.

Skumanich A, Lites BW. 1987. Stokes profile analysis and vector magnetic fields. I - Inversion of photospheric lines. Astrophys $J$ 322: 473-482. https://doi.org/10.1086/165743.

Smith CM. 1904. Widened lines in sunspot spectra. Kodaik Obs Bull 1: $1-11$.

Solanki SK, Wenzler T, Schmitt D. 2008. Moments of the latitudinal dependence of the sunspot cycle: A new diagnostic of dynamo models. A\&A 483(2): 623-632. https://doi.org/10.1051/00046361:20054282.

Staude J. 1991. Solar research at potsdam: Papers on the structure and dynamics of sunspots. Rev Mod Astron 4: 69-89. https://doi. org/10.1007/978-3-642-76750-0_5.

Steen O, Maltby P. 1960. On the correlation between observations of magnetic fields of sunspots at Mt. Wilson, Potsdam and the Crimea. Astrophys Norv 6: 263.

Stenflo JO. 1970. Hale's attempts to determine the Sun's general magnetic field. Sol Phys 14(2): 263-273. https://doi.org/10.1007/ BF00221312.

Stenflo JO. 2017. History of solar magnetic fields since George Ellery Hale. Space Sci Rev 210(1-4): 5-35. https://doi.org/ 10.1007/s11214-015-0198-z.

Stenflo JO, Kosovichev AG. 2012. Bipolar magnetic regions on the Sun: Global analysis of the SOHO/MDI data set. Astrophys $J$ 745(2): 129. https://doi.org/10.1088/0004-637X/745/2/129.

Stepanov VE, Severny AB. 1962. A photoelectric method for measurements of the magnitude and direction of the solar magnetic field. Bull Crim Astrophys Obs 28: 166-193.

Svalgaard L, Duvall TL, Scherrer PH. 1978. The strength of the Sun's polar fields. Sol Phys 58(2): 225-239. https://doi.org/ 10.1007/BF00157268.

Thiessen G. 1946. La mesure du champ magnétique général du Soleil. Ann Astrophys 9: 101.

Tlatov AG, Pevtsov AA. 2010. The latitude of ephemeral regions as an indicator for solar-cycle strength. Mem Soc Astron Italiana 81: 814.

Tlatov AG, Pevtsov AA. 2014. Bimodal distribution of magnetic fields and areas of sunspots. Sol Phys 289(4): 1143-1152. https://doi.org/10.1007/s11207-013-0382-9.

Tlatov AG, Tlatova KA, Vasil'eva VV, Pevtsov AA, Mursula K. 2015. Properties of sunspot umbrae of leading and trailing polarity in 1917-2013. Adv Space Res 55(3): 835-842. https://doi.org/ 10.1016/j.asr.2014.05.033. 
Tlatova K, Tlatov A, Pevtsov A, Mursula K, Vasil'eva V, Heikkinen E, Bertello L, Pevtsov A, Virtanen I, Karachik N. 2018. Tilt of sunspot bipoles in solar cycles 15 to 24. Sol Phys 293(8): 118. https://doi.org/10.1007/s11207-018-1337-y.

Tlatov A, Riehokainen A, Tlatova K. 2019. The characteristic sizes of the sunspots and pores in solar cycle 24. Sol Phys 294(4): 45. https://doi.org/10.1007/s11207-019-1439-1.

Tsuneta S, Ichimoto K, Katsukawa Y, Lites BW, Matsuzaki K, et al. 2008. The magnetic landscape of the Sun's polar region. Astrophys $J$ 688(2): 1374-1381. https://doi.org/10.1086/592226.

Ulrich RK. 2001. Very long lived wave patterns detected in the solar surface velocity signal. Astrophys $J$ 560(1): 466-475. https://doi. org/10.1086/322524.

Ulrich RK, Boyden JE. 2006. Carrington coordinates and solar maps. Sol Phys 235(1-2): 17-29. https://doi.org/10.1007/s11207-0060041-5.

van Driel-Gesztelyi L, Green LM. 2015. Evolution of active regions. Living Rev Sol Phys 12(1): 1. https://doi.org/10.1007/lrsp-2015-1.

Virtanen I, Mursula K. 2017. Photospheric and coronal magnetic fields in six magnetographs. II. Harmonic scaling of field intensities. $A \& A$ 604: A7. https://doi.org/10.1051/0004-6361/201730863.

Virtanen IOI, Virtanen II, Pevtsov AA, Yeates A, Mursula K. 2017. Reconstructing solar magnetic fields from historical observations. II. Testing the surface flux transport model. A\&A 604: A8. https://doi.org/10.1051/0004-6361/201730415.

Virtanen II, Pevtsov AA, Mursula K. 2019a. Structure and evolution of the photospheric magnetic field in 2010-2017: comparison of SOLIS/VSM vector field and BLOS potential field. A\&A $\mathbf{6 2 4}$ : A73. https://doi.org/10.1051/0004-6361/201834895.

Virtanen IOI, Virtanen II, Pevtsov AA, Mursula K. 2019b. Reconstructing solar magnetic fields from historical observations. VI. Axial dipole moments of solar active regions in cycles 21-24. A\&A 632: A39. https://doi.org/10.1051/0004-6361/201936134.
Vitinsky YI, Kopecký M, Kuklin GV. 1986. The statistics of sunspots (Statistika pjatnoobrazovatelnoj dejatelnosti solntsa), Nauka, Moscow. (In Russian).

von Klüber H. 1948. Zum Nachweis schwacher Magnetfelder auf der Sonnenberfläche. Mit 8 Textabbidungen. ZAp 24: 1 .

Wallace RJ. 1907. Studies in sensitometry II orthochromatism by bathing. Astrophys J 26: 299. https://doi.org/10.1086/141509.

Watson FT, Fletcher L, Marshall S. 2011. Evolution of sunspot properties during solar cycle 23. A\&A 533: A14. https://doi.org/ 10.1051/0004-6361/201116655.

Whitbread T, Yeates AR, Muñoz-Jaramillo A, Petrie GJD. 2017. Parameter optimization for surface flux transport models. $A \& A$ 607: A76. https://doi.org/10.1051/0004-6361/201730689.

Worden J, Harvey J. 2000. An evolving synoptic magnetic flux map and Implications for the distribution of photospheric magnetic flux. Sol Phys 195(2): 247-268. https://doi.org/10.1023/ A: 1005272502885 .

Yeates AR. 2020. The minimal helicity of solar coronal magnetic fields. Astrophys J 898: L49. https://doi.org/10.3847/2041-8213/ aba762.

Young CA. 1895. The sun. In: 34 of International scientific series. [American ed.], D. Appleton and Company, new and revised edn, 375 p. ADS: https://ui.adsabs.harvard.edu/abs/1895sun..book..... Y/abstract.

Zeeman P. 1897. On the influence of magnetism on the nature of the light emitted by a substance. Astrophys J 5: 332. https://doi.org/ $10.1086 / 140355$.

Zeeman P. 1913. Researches in magneto-optics. MACMILLAN and CO, London.

Zirin H. 1988. Astrophysics of the sun. Cambridge University Press, Cambridge, UK. ISBN 0521302684. 